\title{
Digital in-line holography assessment for general phase and opaque particle
}

\author{
S. Coëtmellec \\ coetmellec@coria.fr \\ W. Wichitwong \\ G. Gréhan \\ D. Lebrun
}

\section{Brunel}

\section{A. J. E. M. Janssen}

Département d'optique, UMR-6614, Av. de l’Université, 76801 Saint-Etienne du Rouvray cedex, France

Département d'optique, UMR-6614, Av. de l’Université, 76801 Saint-Etienne du Rouvray cedex, France

Département d'optique, UMR-6614, Av. de l’Université, 76801 Saint-Etienne du Rouvray cedex, France

Département d'optique, UMR-6614, Av. de l'Université, 76801 Saint-Etienne du Rouvray cedex, France

We propose using the circle polynomials to describe a particle's transmission function in a digital holography setup. This allows both opaque and phase particles to be determined. By means of this description, we demonstrate that it is possible to estimate the digital in-line hologram produced by a spherical particle. The experimental intensity distribution due to an opaque micro-inclusion is compared to the theoretical one obtained by our new model. Moreover, the simulated hologram and reconstructed image of the particle by an optimal fractional Fourier transformation under the opaque disk, quadratic phase, and quasi-spherical phase approximation are compared with the results obtained by simulating holograms by the Lorenz-Mie Theory (LMT). The Zernike coefficients corresponding to the considered particles are evaluated using the double exponential (DE) method which is optimal in various respects.

[DOI: http://dx.doi.org/10.2971/jeos.2014.14021]

Keywords: Digital in-line holography, Lorenz-Mie theory, double exponential formula, Zernike coefficients, inclusion

\section{INTRODUCTION}

This work is motivated by the fact that in studies on digital holography a spherical particle is generally considered as an opaque disk, not as a spherical object or, even better, as a thin lens. Under the opaque disk approximation, the refractive index is not taken into account. Furthermore, a particle can be purely absorbent, such as metal particles, but it can instead be transparent, such as glass particles or water droplets. Recently, some considerations about phase objects have been given as to whether it is possible to interpret a particle as a phase object [1]-[4]. Based on these findings, it is felt that a different interpretation is required. In the field of particle holography, it is generally assumed that an in-line hologram is only due to the edge of the particle, which justifies the opaque disk approximation. The part of the beam diffracted by the edge of the particle interferes with the other part of the beam when creating the hologram. Then, under this approximation, the refractive index of the particle is not taken into account in the imaging model. It should also be noted that the digital reconstruction of the image of the object does not put in evidence the spot of light at the center of the reconstructed image predicted by the Lorenz-Mie theory (LMT). In this paper, we propose a novel view by incorporating the inside region of the particle using a thickness function. Hence, we present, firstly, a general expression for the intensity distribution in the plane of the CCD sensor. The theoretical background for this is based on two tools: the ABCD matrix formalism and circle polynomial decompositions. The fractional Fourier transformation is used to reconstruct the image of the particle and the optimal fractional order is deduced from the ABCD matrix formalism. Although this is not contained in the publication, the major advantage of the circle polynomials to describe the particle from the reconstruction by means of the fractional Fourier transformation point of view is that when the image of the object is reconstructed, the image function is described again by Zernike polynomials. Note again that this point is not presented here. In this paper, we focused on the forward problem even if we show that the fractional Fourier transformation is used to reconstruct the image of the particle. In the second and third parts, the theoretical model is compared to two configurations, viz., an experimental one involving water droplets and a theoretical one involving a numerical approach based on the rigorous near-field Lorenz-Mie scattering theory. The experimental configuration involves inclusion in a water droplet. The included object here is an opaque polyethylene microsphere. This experimental context is very important in many areas. We define an inclusion as one foreign body in another. In biology, inclusion corresponds to an intracellular substance not belonging to the normal con- 
stituents of the cell, like droplets containing bacterial cells or other entities. This is important in terms of the optical detection of these foreign bodies, where biological organisms can survive for prolonged periods when contained within a protective liquid coating [5]. This situation may be encountered in the cooling towers of nuclear power plants, for example, or in any wet environment at high temperatures. Another example, water droplets in the atmosphere that contain insoluble inclusions, is of essential interest for climatology [6]. The knowledge of the removal of submicron aerosol particles from the dispersion of radionuclides by washout is very important in the context of public health [7]. In physics, inclusions are intensively study by the Lorenz-Mie theory to describe the morphology-dependent resonances (MDRs) [8]. Next, the second configuration is a theoretical configuration where a plane wave illuminates a spherical water droplet. This configuration permits us to use the Lorenz-Mie theory. In this theory, the wave must be plane, unlike in the Generalized Lorenz-Mie Theory (GMTL), where we can have a Gaussian beam. This part is theoretical rather than experimental because it simplifies the control of the parameters of the optical system. At the end of the paper, we propose a numerical method to evaluate the Zernike moments by means of the double exponential formula.

\section{GENERAL CONFIGURATION OF THE OPTICAL SYSTEM IN DIGITAL IN-LINE HOLOGRAPHY}

It should be noted that most optical systems for digital in-line holography are composed of two parts. Each part is delimited by the source, the object (in our case the object is a particle) and the CCD sensor. An illustration is given in Figure 1. The two parts can be fully described by two $4 \times 4$ symplectic matrices, denoted $\mathbb{M}_{i}$ for the system between the source (waist $\omega_{0}$ ) and the particle, and denoted $\mathbb{M}_{t}$ between the particle and the CCD sensor. Each symplectic matrix is composed of the $2 \times 2$ matrices $\mathbb{A}, \mathbb{B}, \mathbb{C}$ and $\mathbb{D}[10]-[11]$.

Then $\mathbb{M}_{i}$ and $\mathbb{M}_{t}$ are defined as

$$
\mathbb{M}_{i}:=\left(\begin{array}{cc}
\mathbb{A}_{i} & \mathbb{B}_{i} \\
\mathbb{C}_{i} & \mathbb{D}_{i}
\end{array}\right), \quad \quad \mathbb{M}_{t}:=\left(\begin{array}{cc}
\mathbb{A}_{t} & \mathbb{B}_{t} \\
\mathbb{C}_{t} & \mathbb{D}_{t}
\end{array}\right)
$$

Symplecticity of the matrix $\mathbb{M}$ is defined in [42], Eq. (5) on page 919 . Here, the matrix $\mathbb{M}_{i}$ takes the following form:

$$
\begin{array}{ll}
\mathbb{A}_{\mathrm{i}}=\left(\begin{array}{cc}
a_{\mathrm{i}, 1} & 0 \\
0 & a_{\mathrm{i}, 2}
\end{array}\right), & \mathbb{B}_{\mathrm{i}}=\left(\begin{array}{cc}
b_{\mathrm{i}, 1} & 0 \\
0 & b_{\mathrm{i}, 2}
\end{array}\right), \\
\mathbb{C}_{\mathrm{i}}=\left(\begin{array}{cc}
c_{\mathrm{i}, 1} & 0 \\
0 & c_{\mathrm{i}, 2}
\end{array}\right), & \mathbb{D}_{\mathrm{i}}=\left(\begin{array}{cc}
d_{\mathrm{i}, 1} & 0 \\
0 & d_{\mathrm{i}, 2}
\end{array}\right),
\end{array}
$$

where $a_{i, j}, b_{i, j}, c_{i, j}$ and $d_{\mathrm{i}, j}$ are defined by the optical components. The subscript $i$ indicates the incident part and $j$ corresponds to the transverse coordinates perpendicular to the optical axis. The subscripts 1 and 2 indicate the horizontal axis and the vertical axis, respectively. The value of the diagonal elements correspond to the cases where the system is an anamorphic or a circular optical system. In the same way, $\mathbb{M}_{t}$

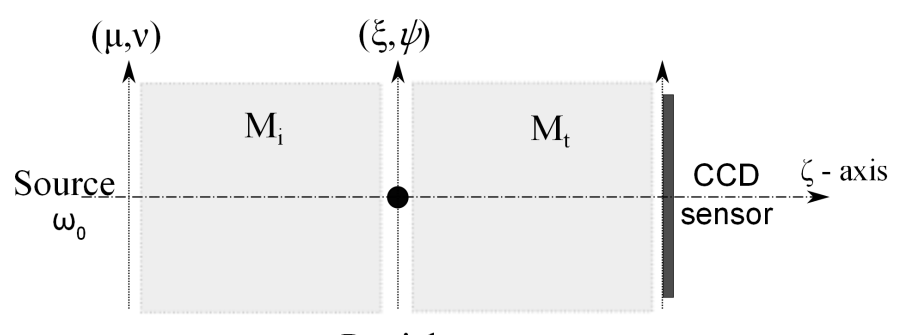

Particle

FIG. 1 Digital in-line holography setup: source $\lambda$, matrix $M_{i}$, illuminated-object, matrix $M_{t}$ and CCD sensor

takes the following form:

$$
\begin{array}{ll}
\mathbb{A}_{t}=\left(\begin{array}{cc}
a_{t, 1} & 0 \\
0 & a_{t, 2}
\end{array}\right), & \mathbb{B}_{t}=\left(\begin{array}{cc}
b_{t, 1} & 0 \\
0 & b_{t, 2}
\end{array}\right), \\
\mathbb{C}_{t}=\left(\begin{array}{cc}
c_{t, 1} & 0 \\
0 & c_{t, 2}
\end{array}\right), & \mathbb{D}_{t}=\left(\begin{array}{cc}
d_{t, 1} & 0 \\
0 & d_{t, 2}
\end{array}\right),
\end{array}
$$

where $a_{t, j}, b_{t, j}, c_{t, j}$ and $d_{t, j}$ are defined by the optical components from the particle plane to the CCD sensor. From Figure 1, the propagation of the Gaussian beam from the first waist $\omega_{0}$ to the plane of the CCD sensor can be described by two linear canonical transformations [13]. Each linear canonical transformation, denoted $\mathcal{C}$, has the same integral structure and for the first part, it is given by

$$
\begin{aligned}
& \mathcal{C}\left[G_{0}(\boldsymbol{\rho})\right](\mathbf{r})=\frac{\exp \left(i k E_{i}\right)}{i \lambda \sqrt{\operatorname{det}\left(\mathbb{B}_{i}\right)}} \int_{\mathbb{R}^{2}} G_{0}(\boldsymbol{\rho}) \\
& \quad \cdot \exp \left[i \frac{\pi}{\lambda}\left(\boldsymbol{\rho}^{T} \mathbb{B}_{i}^{-1} \mathbb{A}_{i} \boldsymbol{\rho}-2 \boldsymbol{\rho}^{T} \mathbb{B}_{i}^{-1} \mathbf{r}+\mathbf{r}^{T} \mathbb{D}_{i} \mathbb{B}_{i}^{-1} \mathbf{r}\right)\right] d \boldsymbol{\rho} .
\end{aligned}
$$

with $\lambda$ the wavelength and $k=2 \pi / \lambda$. Now, in the plane of the first waist (the first waist is referred to as $\omega_{0}$ ), at the left hand side of Figure 1, the amplitude of the Gaussian beam, denoted by $G_{0}$, is defined as

$$
G_{0}(\rho)=\exp \left(-\rho^{T} \mathbb{Q}_{0}^{-1} \rho\right),
$$

where $\boldsymbol{\rho}=(\mu v)^{T}$ and the real matrix $\mathbb{Q}_{0}^{-1}$ stands for the Gaussian beam width $\omega_{0}$ given by

$$
\mathbf{Q}_{0}=\left(\begin{array}{cc}
\omega_{0}^{2} & 0 \\
0 & \omega_{0}^{2}
\end{array}\right)
$$

By means of [14]-[16], the amplitude of the incident Gaussian beam, denoted by $G_{i}(\mathbf{r})$, just before reaching the particle, is given by

$$
G_{i}(\mathbf{r})=\frac{\pi}{i \lambda} \frac{\exp \left(i k E_{i}\right)}{\sqrt{\operatorname{det}\left(\mathbb{B}_{i} \mathbb{Q}_{0}^{-1}-i \frac{\pi}{\lambda} \mathbb{A}_{i}\right)}} \cdot \exp \left[-\frac{\pi^{2}}{\lambda^{2}} \mathbf{r}^{T} \mathbf{Q}_{i}^{-1} \mathbf{r}\right]
$$

with

$$
\mathbb{Q}_{i}^{-1}=\mathbb{B}_{i}^{-1 T}\left(\mathbb{Q}_{0}^{-1}-i \frac{\pi}{\lambda} \mathbb{B}_{i}^{-1} \mathbb{A}_{i}\right)^{-1} \mathbb{B}_{i}^{-1}-i \frac{\lambda}{\pi} \mathbb{D}_{i} \mathbb{B}_{i}^{-1},
$$

and the position vector in the transverse plane $\mathbf{r}^{T}=(\xi \psi)$. The $\mathcal{L}_{2}$-norm is used by noting that $|\mathbf{r}|=r=\sqrt{\xi^{2}+\psi^{2}}$. The distance $E_{i}$ depends on the optical paths, each weighted by the index of the medium traversed by the beam along that path. Now, from the point of view of the definition of a particle, most of the literature on digital holography assumes that the 
particle is a pure amplitude object and in the definition of the particle function, denoted $[1-p]$, the pupil $p$ is expanded into a chirped Gaussian series as in [17]-[21]:

$$
p=\sum_{k=1}^{N} A_{k} \exp \left(-r^{T} \cdot R^{T} P_{k} R \cdot r\right),
$$

where the matrices $R$ and $P_{k}$ can be found in [18]. This choice of definition, Eq. (9), is in order to simplify the calculations, because it involves Gaussian functions and is of interest for elliptic particles. However, particles are generally described by a complicated phase function. Therefore, in this paper, we wish to extend the pupil function into a Zernike series as in [22], Eq. (6). This choice makes it possible to develop the cases of an opaque or a phase particle. Consequently, we assume that we have expanded the generalized complex pupil function $p(s, \theta)$ as

$$
\begin{aligned}
p(s, \theta) & =[1-A(s, \theta) \cdot \exp (i \Phi(s, \theta))] \cdot Z_{0}^{0}(s, \theta) \\
& =\left[1-\sum_{n, m} \gamma_{n}^{m} \cdot Z_{n}^{m}(s, \theta)\right] Z_{0}^{0}(s, \theta)
\end{aligned}
$$

for a phase particle. The summation range for $n$ is from zero to infinity and the summation over $m$ is performed from $-n$ to $n$ with $n-|m|$ even. The circle polynomials, denoted here by $Z_{n}^{m}$ in Eq. (10), are given for $m, n$ and $n-|m|$ even and non-negative by

$$
Z_{n}^{m}(s, \theta)=R_{n}^{|m|}(s) \cdot e^{i m \theta}, \quad 0 \leq s \leq 1, \quad 0 \leq \theta \leq 2 \pi,
$$

and the Zernike coefficients $\gamma_{n}^{m}$ are obtained by using the orthogonality of the Zernike circle polynomials $Z_{n}^{m}$, so that

$$
\begin{aligned}
& \gamma_{n}^{m} \\
& =\frac{n+1}{\pi} \int_{0}^{1} \int_{0}^{2 \pi} A(s, \theta) \exp [i \Phi(s, \theta)] \cdot \overline{Z_{n}^{m}(s, \theta)} s d s d \theta .
\end{aligned}
$$

A bar over a quantity indicates complex conjugation. In the case of an opaque particle, the pupil function $p$ corresponds to the particular case where the amplitude $A(s, \theta)$ is equal to unity and the phase $\Phi(s, \theta)$ is equal to zero, and then

$$
p(s, \theta)=\gamma_{0}^{0} \cdot Z_{0}^{0}(s, \theta),
$$

with $\gamma_{0}^{0}=1$. In the plane of the CCD sensor, the field's amplitude, denoted by $G_{\mathcal{C}}\left(\mathbf{r}^{\prime}\right)$, is obtained by means of the linear canonical integral,

$$
\begin{aligned}
G_{c}\left(\mathbf{r}^{\prime}\right)= & \frac{\exp \left(i k E_{t}\right)}{i \lambda \sqrt{\operatorname{det}\left(\mathbb{B}_{t}\right)}} \exp \left[i \frac{\pi}{\lambda} \mathbf{r}^{T} \mathbb{B}_{t}^{-1} \mathbb{D}_{t} \mathbf{r}^{\prime}\right] \\
& \times \int_{\mathbb{R}^{2}} G_{i}(\mathbf{r}) \cdot[1-p(s, \theta)] \exp \left[i \frac{\pi}{\lambda} \mathbf{r}^{T} \mathbb{B}_{t}^{-1} \mathbb{A}_{t} \mathbf{r}\right] \\
& \times \exp \left[-i \frac{2 \pi}{\lambda} \mathbf{r}^{T} \mathbb{B}_{t}^{-1} \mathbf{r}^{\prime}\right] d \mathbf{r},
\end{aligned}
$$

with $\mathbb{A}_{t}, \mathbb{B}_{t}, \mathbb{C}_{t}, \mathbb{D}_{t}$ as given in Eq. (3). To normalize the integral in Eq. (14), the dimensionless variables $\mathbf{s}=2 \mathbf{r} / D$ and $\mathbf{s}^{\prime}=2 \mathbf{r}^{\prime} / D$ with $D$ the diameter of the particle can be introduced. Then, Eqs. (7) and (14) can be combined to produce

$$
\begin{aligned}
\widehat{G}_{c}\left(\mathbf{s}^{\prime}\right)=- & \frac{\pi D^{2}}{4 \lambda^{2}} \frac{\exp \left[i k\left(E_{i}+E_{t}\right)\right]}{\sqrt{\operatorname{det}\left(\mathbb{B}_{t}\right) \operatorname{det}\left(\mathbb{B}_{i} \mathbb{Q}_{0}^{-1}-i \frac{\pi}{\lambda} \mathbb{A}_{i}\right)}} \\
& \times \exp \left[i \frac{\pi D^{2}}{4 \lambda} \mathbf{s}^{\prime T} \mathbb{D}_{t} \mathbb{B}_{t}^{-1} \mathbf{s}^{\prime}\right] \cdot \int_{\mathbb{R}^{2}}[1-p(s, \theta)] \\
& \times \exp \left[i \mathbf{s}^{T} \mathbb{L}_{t} \mathbf{s}\right] \exp \left[-i 2 \pi \mathbf{s}^{T} \mathbb{P}_{t} \mathbf{s}^{\prime}\right] d \mathbf{s},
\end{aligned}
$$

with $\widehat{G}_{\mathcal{C}}\left(\mathbf{s}^{\prime}\right)=G_{c}\left((D / 2) \mathbf{s}^{\prime}\right)$ and

$$
\mathbb{L}_{t}=\frac{\pi D^{2}}{4 \lambda} \mathbb{B}_{t}^{-1} \mathbb{A}_{t}+i\left(\frac{\pi D}{2 \lambda}\right)^{2} \mathbb{Q}_{i}, \quad \mathbb{P}_{t}=\frac{D^{2}}{4 \lambda} \cdot \mathbb{B}_{t}^{-1} .
$$

To simplify the calculations, we introduce $\sigma=\mathbb{P}_{t} \mathbf{s}^{\prime}$. With this change of variables, Eq. (15) takes the form

$$
\begin{aligned}
\widehat{\widehat{G}}_{c}(\sigma) & =-\frac{\pi D^{2}}{4 \lambda^{2}} \\
\cdot & \frac{\exp \left[i k\left(E_{i}+E_{t}\right)\right]}{\sqrt{\operatorname{det}\left(\mathbb{B}_{t}\right) \operatorname{det}\left(\mathbb{B}_{i} \mathbf{Q}_{0}^{-1}-i \frac{\pi}{\lambda} \mathbb{A}_{i}\right)}} \exp \left[i \frac{\pi D^{2}}{4 \lambda} \sigma^{T} \mathbf{S}_{t} \sigma\right] \\
& \cdot \int_{\mathbb{R}^{2}}[1-p(s, \theta)] \exp \left[i \mathbf{s}^{T} \mathbb{L}_{t} \mathbf{s}\right] \exp \left[-i 2 \pi \mathbf{s}^{T} \sigma\right] d \mathbf{s},
\end{aligned}
$$

with $\widehat{\widehat{G}}_{c}(\sigma)=\widehat{G}_{c}\left(\mathbb{P}_{t}^{-1} \sigma\right)$ and $S_{t}=\mathbb{P}_{t}^{-1 T} \mathbb{D}_{t} \mathbb{B}_{t}^{-1} \mathbb{P}_{t}^{-1}$. Now, the intensity distribution in the plane of the CCD sensor, denoted $I(\sigma)$, depends on the particle. We can write the intensity as

$$
\begin{aligned}
I(\sigma) & =\left|\widehat{\widehat{G}}_{c}(\sigma)\right|^{2} \\
& =\frac{\pi D^{2}}{4 \lambda^{2}} \frac{\left|G(\sigma)-G_{0}^{0}(\sigma)+\epsilon G_{n}^{m}(\sigma)\right|^{2}}{\mid \sqrt{\operatorname{det}\left(\mathbb{B}_{t}\right) \operatorname{det}\left(\mathbb{B}_{i} \mathbb{Q}_{0}^{-1}-i \frac{\pi}{\lambda} \mathbb{A}_{i}\right)}},
\end{aligned}
$$

with the two following functions

$$
G(\sigma)=\int_{\mathbb{R}^{2}} \exp \left[i \mathbf{s}^{T} \mathbb{L}_{t} \mathbf{s}\right] \exp \left[-i 2 \pi \mathbf{s}^{T} \boldsymbol{\sigma}\right] d \mathbf{s},
$$

and

$$
\begin{aligned}
G_{n}^{m}(\boldsymbol{\sigma})=\sum_{n, m} & \gamma_{n}^{m} \times \int_{\mathbb{R}^{2}} Z_{0}^{0}(s, \theta) Z_{n}^{m}(s, \theta) \\
& \times \exp \left[i \mathbf{s}^{T} \mathbb{L}_{t} \mathbf{s}\right] \exp \left[-i 2 \pi \mathbf{s}^{T} \boldsymbol{\sigma}\right] d \mathbf{s} .
\end{aligned}
$$

The value of $\epsilon$ in Eq. (18) depends on the particle considered. For $\epsilon=0$, the particle is an opaque object and for $\epsilon=1$, it is a phase object. The $G_{n}^{m}$ functions in Eq. (20) are expressed in terms of the standard Cartesian coordinates $\sigma$. It turns out that this integral simplifies when we change the variables $\mathbf{s}$ and $\sigma$ to polar coordinates. By noting that $\sigma=\sigma \exp (i \varphi)$ and $s=s \exp (i \theta)$, we have

$$
\begin{aligned}
G_{n}^{m}(\sigma, \varphi)=\sum_{n, m} & \gamma_{n}^{m} \times \int_{0}^{1} \int_{0}^{2 \pi} Z_{n}^{m}(s, \theta) \exp \left[i \mathbf{s}^{T} \mathbb{L}_{t} \mathbf{s}\right] \\
& \times \exp [-i 2 \pi \sigma \cdot s \cos (\theta-\varphi)] s d s d \theta
\end{aligned}
$$

\subsection{Theoretical developments of $G$ and $G_{n}^{m}$}

The theoretical development of the integral $G(\sigma)$ in Eq. (19) is straightforward. From [13], Eq. (2.303) on p. 57, it follows that it is given by

$$
G(\sigma)=\frac{i \pi}{\sqrt{\operatorname{det}\left(\mathbb{L}_{t}\right)}} \cdot \exp \left[-i \pi^{2} \sigma^{T} \mathbb{L}_{t}^{-1} \sigma\right]
$$

To derive a semi-analytic expression for (21), it is necessary to use mathematical results on (i) a special circle polynomial expansion, (ii) the linearization of products of circle polynomials [23], and (iii) the extended Nijboer-Zernike theory (ENZ) [24]. The details of the computations are given in 
Appendix B. The semi-analytical expression for the integral $G_{n}^{m}(\sigma, \varphi)$ is then

$$
\begin{aligned}
G_{n}^{m}(\sigma, \varphi)=2 & \pi \sum_{q=-\infty}^{+\infty} \sum_{n, m, p}^{\infty} \sum_{t} \gamma_{n}^{m} \cdot(-i)^{m+q} \cdot A_{n,|2 q|+2 p, t}^{m, 2 q, m+2 q} \\
\cdot & \beta_{|2 q|+2 p}^{|2 q|}(\delta) \cdot V_{t}^{m+2 q}(2 \pi \sigma, \chi) \cdot e^{i(m+2 q) \varphi},
\end{aligned}
$$

The summation range over $t$ is

$$
t=\max (|m+2 q|,|m-| 2 q|-2 p|)(2)(n+|2 q|+2 p)
$$

where $\mathrm{a}(2) \mathrm{b}$ denotes $a, a+2, \ldots, b$ when $b-a$ is non-negative and even. The parameter $\chi$ in Eq. (23) is the trace of the matrix $\mathbb{L}_{t}$, so that $\chi=\frac{1}{2} \cdot \operatorname{Tr}\left(\mathbb{L}_{t}\right)$. The $\delta$-parameter is linked to the ellipticity of the system, i.e., the optical components and the droplet. It is defined by $\delta=\frac{1}{2}\left(\mathbb{L}_{t(1,1)}-\mathbb{L}_{t(2,2)}\right)$ where $\mathbb{L}_{t(i, j)}$ are the diagonal elements of $\mathbb{L}_{t}$ in Eq. (16). In the particular case where the optical setup is circular, we have $\delta=0$ and $\beta_{|2 q|+2 p}^{|2 q|}(0)=1$ if $q=p=0$, and 0 otherwise. The coefficients $\beta$ can be expressed explicitly in terms of the hypergeometric functions ${ }_{2} F_{3}$ as in [26], Eqs. (A-11)-(A-13). From [25], Eq. (142), the numbers $A$ in Eq. (23) are related to the ClebschGordon coefficients:

$$
A_{n,|2 q|+2 p, t}^{m, 2 q, m+2 q}=\left|C_{\frac{m}{2}, \frac{n q}{2}, \frac{|2 q|+2 p}{2}, \frac{m+2 q}{2}}^{2}\right|^{2},
$$

where the C's are the Clebsch-Gordon coefficients. The $V$ functions have the series expression $([26,24,27])$

$$
\begin{aligned}
& V_{n}^{m}(r, f) \\
& \quad=\varepsilon_{m} \exp (\text { if }) \cdot \sum_{l=1}^{\infty}(-2 i f)^{l-1} \sum_{j=0}^{P} v_{l j} \cdot \frac{J_{|m|+l+2 j}(r)}{l(r)^{l}},
\end{aligned}
$$

where $n$ and $|m|$ are integers $\geq 0$ with $n-|m|$ even and nonnegative, and

$$
\begin{array}{r}
v_{l j}=(-1)^{P}(|m|+l+2 j)\left(\begin{array}{c}
|m|+j+l-1 \\
l-1
\end{array}\right) \\
\cdot\left(\begin{array}{c}
j+l-1 \\
l-1
\end{array}\right)\left(\begin{array}{c}
l-1 \\
P-j
\end{array}\right) /\left(\begin{array}{c}
Q+l+j \\
l
\end{array}\right),
\end{array}
$$

for $l=1,2, \ldots, j=0,1, \ldots, P, P=\frac{n-|m|}{2}$ and $Q=\frac{n+|m|}{2}$. In Eq. (26), we have to choose $\varepsilon_{m}=-1$ for odd $m<0$ and $\varepsilon_{m}=1$ otherwise. Now, two particular cases can be considered: $\epsilon=0$ so the particle is an opaque object, and $\epsilon=1$ so the particle is a phase object. As we saw earlier, from Eq. (18), the intensity involves only the two functions $G_{n}^{m}$ given in Eq. (20).

\subsection{Reconstruction by a fractional Fourier transformation}

From the digital holography reconstruction point of view, the fractional Fourier transformation (FRFT) is used to reconstruct the image of an object [29]. The FRFT of orders $\left(a_{x}, a_{y}\right) \in[0,1] \times[0,1]$ of an intensity image $I(x, y)$ is defined as $[30,31,32]$

$$
\begin{aligned}
& \mathcal{F}_{a_{x}, a_{y}}[I(x, y)]\left(x_{a}, y_{a}\right) \\
& \quad=\int_{\mathbb{R}^{2}} N_{a_{x}}\left(x, x_{a}\right) N_{a_{y}}\left(y, y_{a}\right) I(x, y) d x d y,
\end{aligned}
$$

where $s_{j}^{2}=N_{j} \cdot \delta_{j}^{2}, j=x, y$ and the kernel of the fractional operator is

$$
\begin{aligned}
& N_{a_{j}}\left(x, x_{a}\right) \\
& \quad=C\left(a_{j}\right) \exp \left(i \pi \frac{x^{2}+x_{a}^{2}}{s_{j}^{2} \tan \left(\frac{a_{j} \pi}{2}\right)}\right) \exp \left(-\frac{i 2 \pi x_{a} x}{s_{j}^{2} \sin \left(\frac{a_{j} \pi}{2}\right)}\right),
\end{aligned}
$$

and

$$
C\left(a_{j}\right)=\frac{\exp \left[-i\left(\frac{\pi}{4} \operatorname{sign}\left(\sin \frac{a_{j} \pi}{2}\right)-\frac{a_{j} \pi}{4}\right)\right]}{\left|s_{j}^{2} \sin \frac{a_{j} \pi}{2}\right|^{1 / 2}} .
$$

FRFT is a mathematical tool which is the best operator to analyze linear chirps from signal processing point of view. Moreover the linear chirps can be different along two orthogonal axis. This point is due to the fact that the kernel is separable. Fresnel's integral is although a mathematical operator but its ultima aim is to describe the propagation. Although it could be used to analyze all types of optical signals (here the holograms), the interpretation and the justification to take a separable kernel is difficult because all parameters in the Fresnel's integral have a physical senses. With the FRFT we have not this such problem and specially when we have an astigmatic beam. Now, the image of the particle can be reconstructed with the real optimal fractional orders, denoted here by $a_{x o}$ and $a_{y o}$. To do this, the following conditions must be satisfied:

$$
\begin{aligned}
& \cot \left[\frac{a_{x o} \pi}{2}\right]=-\frac{s_{x}^{2}}{\pi} \cdot \Re\left\{\left[\phi_{11}\right]\right\} \quad \text { and } \\
& \cot \left[\frac{a_{y o} \pi}{2}\right]=-\frac{s_{y}^{2}}{\pi} \cdot \Re\left\{\left[\phi_{22}\right]\right\},
\end{aligned}
$$

where $\left[\phi_{k l}\right]$ is the $2 \times 2$-matrix defined by

$$
\left[\phi_{k l}\right]=-\frac{\pi D^{2}}{4 \lambda} \cdot \mathbb{B}_{t}^{-1^{T}} \cdot \overline{\mathbb{L}_{t}^{-1}} \cdot \mathbb{B}_{t}^{-1},
$$

with $(k, l) \in[1,2] \times[1,2]$.

\section{HOLOGRAMS OF AN OPAQUE PARTICLE AS AN INCLUSION}

In this section, we have chosen to compare the above theoretical developments with some experimental results. The experimental setup of interest is represented in Figure 2. The incident Gaussian beam propagates across an optical system to illuminate a water droplet (with refractive index equal to $n=1.33$ ) of diameter $d$. Inside the droplet, spherical particles of diameter $D$ are added to create the inclusion. In the theoretical developments, the finite droplet size is not taken into account. On the other hand, the radii of curvatures are only

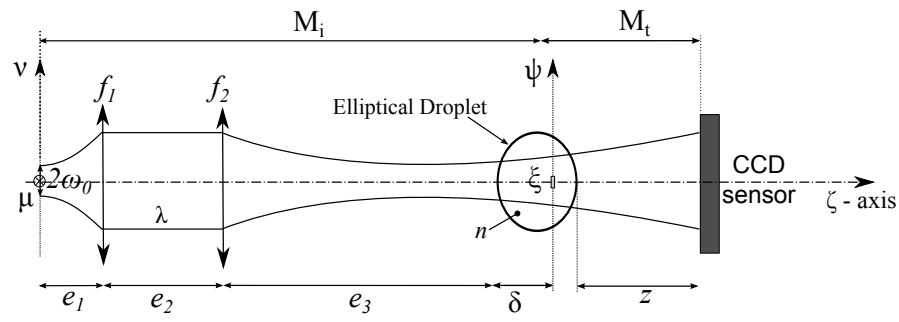

FIG. 2 Numerical and experimental optical setup for $\lambda=642 \mathrm{~nm}, \omega_{0}=2.5 \mu \mathrm{m}$, $f_{1}=50.4 \mathrm{~mm}, f_{2}=5.5 \mathrm{~mm}, e_{1}=f_{1}$ 


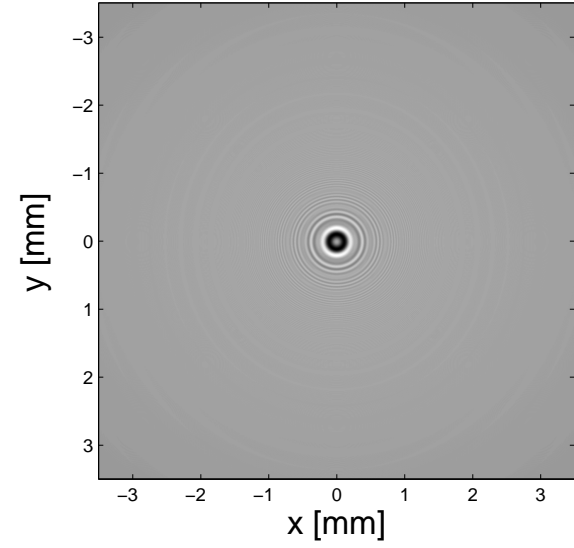

(a)

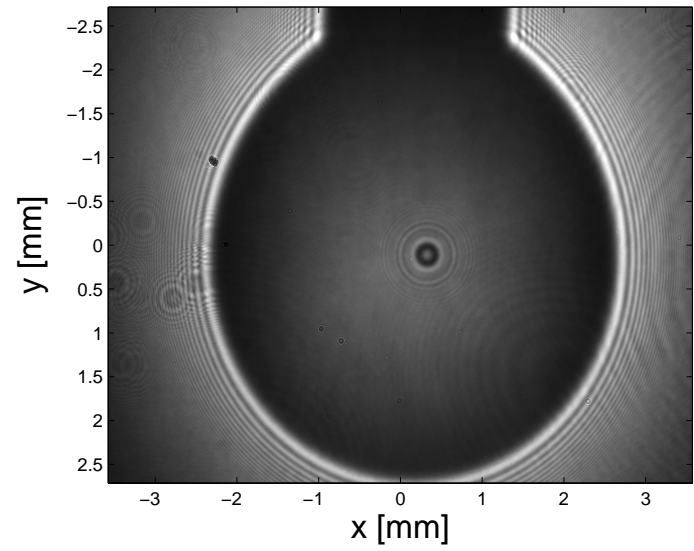

(b)

FIG. 3 Hologram of an opaque micro-inclusion in a droplet obtained from (a) theoretical development and (b) experimental results

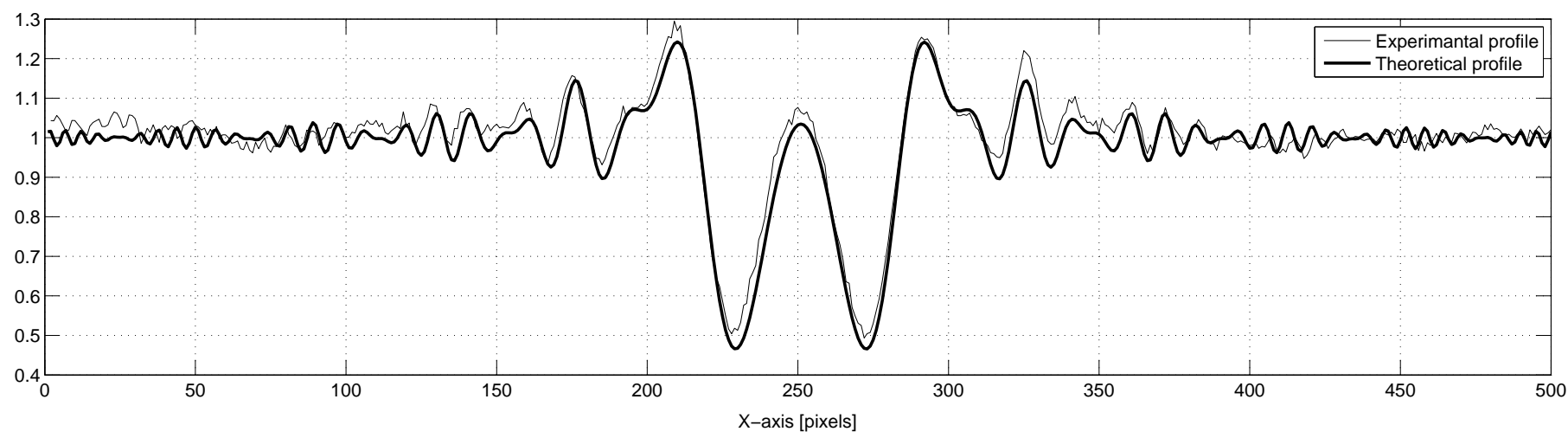

(a)

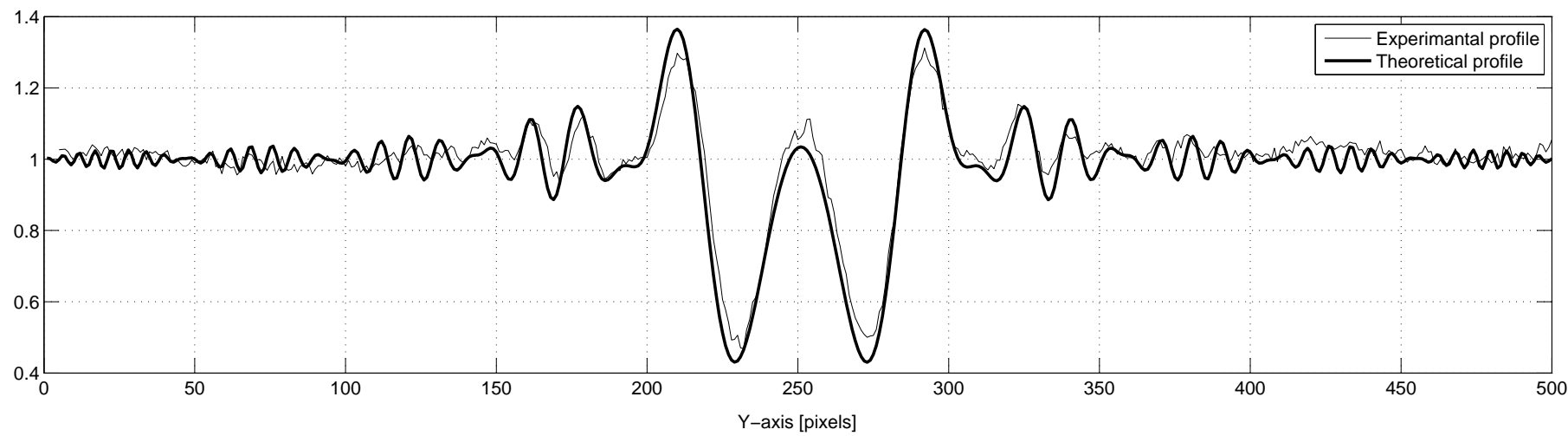

(b)

FIG. 4 Simulated and experimental intensity profiles from the holograms in Figure 3 with $D=23.5 \mu \mathrm{m}, \lambda=642 \mathrm{~nm}, z=6.1 \mathrm{~mm}$.

involved in the model. Behind the droplet, the CCD sensor is located at a distance $z$ to record the intensity distribution of the interference pattern. Note here that the distance $E_{i}$ in Eq. (4) is equal to $E_{i}=e_{1}+e_{2}+e_{3}+n \delta$.

\subsection{The case of a circular system}

In the case of an opaque particle, i.e., for $n=m=0$ and a circular system, i.e., $\delta=0, G_{n}^{m}$ takes the following form:

$$
G_{0}^{0}(\sigma, \varphi)=2 \pi \cdot V_{0}^{0}(2 \pi \sigma, \chi),
$$

with $A_{0,0, t}^{0,0,0}=1$ for all $t$ in Eq. (24). This case is only a particular case of an anamorphic optical system. Due to gravity, the droplet is not spherical and the diameters along two perpendicular axes are not the same. The following section allows us to make a better comparison between the theoretical and the experimental results.

\subsection{The case of an anamorphic optical system}

In the case of an anamorphic optical system, i.e., $\delta \neq 0, G_{n}^{m}$ is defined by

$$
\begin{aligned}
& G_{0}^{0}(\sigma, \varphi)= \\
& 2 \pi \sum_{q=-\infty}^{+\infty} \sum_{p, t}(-i)^{q} \cdot \beta_{|2 q|+2 p}^{|2 q|}(\delta) \cdot V_{t}^{2 q}(2 \pi \sigma, \chi) \cdot e^{i 2 q \varphi} .
\end{aligned}
$$

Eq. (34) takes exactly the form of Eq. (6) in [33] for a centered particle. An illustration of the theoretical development for an opaque micro-inclusion is given in Figure 3(a).

In Figure 4, the simulated and experimental intensity profiles along the $x$-axis and along the $y$-axis are given. These profiles are obtained after normalization. As one can see, simulation and experiment are in very close agreement. That confirms 


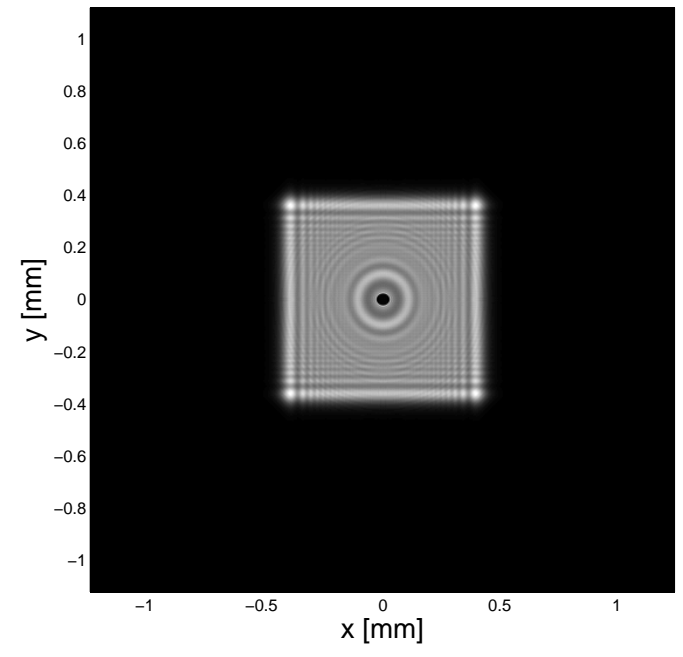

(a)

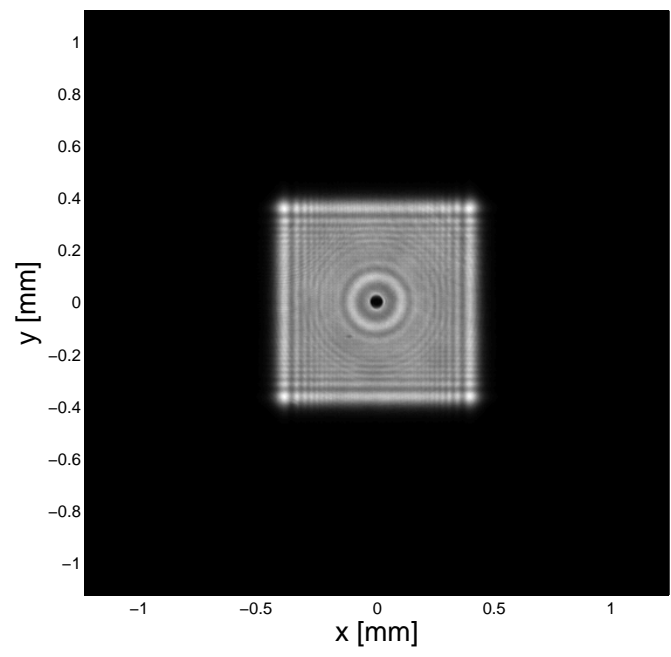

(b)

FIG. 5 Images reconstructed by the fractional Fourier transformation with $a_{x o}=0.766$ and $a_{y_{0}}=0.763$ (a) simulated diffraction pattern of the inclusion (b) experimental diffraction pattern of the inclusion.

the previous theoretical developments. We conclude that the mathematical description of the hologram is valid.

In this simulation, the diameter of the opaque micro-inclusion is $D=23.5 \mu \mathrm{m}$ and its position in the droplet is approximately $\delta=0.99 \mathrm{~mm}$. The radii of curvatures of the droplet are $1.7 \mathrm{~mm}$ along the $x$-axis and $2.3 \mathrm{~mm}$ along the $y$-axis and from the experimental optical setup, the numerical distances are $e_{2}=409.56 \mathrm{~mm}, e_{3}=12 \mathrm{~mm}$ and $z=6.1 \mathrm{~mm}$. With the aim of reconstructing the image of the inclusion, the pictures of the holograms have been cut off at $4.4 \mu \mathrm{m}$ in 1624 by 1234 pixels around the center of the diffraction pattern. The reconstructed inclusion images by means of the FRFT from the simulated diffraction pattern in Figure 3(a) and from the experimental diffraction pattern in Figure 3(b) are shown in Figures 5(a) and 5(b). The optimal fractional orders obtained from Eqs. (32) are $a_{x o}=0.766$ and $a_{y o}=0.763$. The shape of the reconstructed images (i.e., the opaque disk images) are well recovered.

Let us note that when the reconstructed image of an object, here the inclusion, is realized, a scale factor must be applied to retrieve the correct diameter. This point is not developed here but the process is the same as in [34], see Eq. (39). Furthermore, the finite dimensions of the pixels and the coherence here are not taken into account [35].

\section{DIGITAL IN-LINE HOLOGRAMS FOR A SPHERICAL PARTICLE}

In this section, the particle is considered as a droplet in a homogeneous medium. This is a phase particle in the sense that the index and the spherical shape of the droplet is taken into account. Here, we have chosen to compare our holographic model with a general numerical standard of holograms of fields of particles based on the rigorous near-field LorenzMie scattering theory (LMT) [36]. The comparison between the theoretical development proposed in this publication and the near-field Lorenz-Mie scattering theory is based on a particle of diameter $D$ and refractive index $n_{i}$ with the surrounding medium index $n$. In the context of the near-field Lorenz-

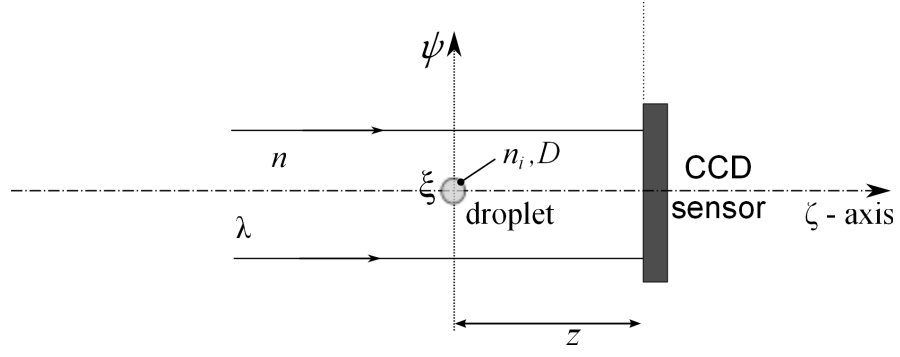

FIG. 6 Configuration under study with $n$ the refractive index of the medium and $n_{i}$ the refractive index of the droplet.

Mie scattering theory, the incident beam on the particle must be a plane wave, something which modifies here only the definition of the ABCD matrices. The configuration under study is illustrated in Figure 6.

The spherical shape of the droplet usually leads to treating the droplet as a thin lens in the same way as a ball-shaped lens does [37]. In the present study, we consider two ways of approximating the droplet. The first is the classical approximation, which is to model the droplet as a thin lens. For the second way, let us recall that in digital holography practice, attention is limited to the edge function defined by the boundaries of the object, while we propose to use the thickness function of the object. The thickness function leads to a variable optical path of the beam inside the droplet in the sense of geometric optics. This means that this point of view remains an approximation compared to the LMT. Each result presented in this article is systematically compared to the other available result, notably the near-field Lorenz-Mie scattering theory. With the near-field Lorenz-Mie scattering theory and the approximation of the droplet as an opaque disk defining the framework of the study, Figure 7 illustrates the simulated holograms for a droplet (i.e., by means of the LMT) and for an opaque disk.

The arrows indicate the positions where the zero crossings of the modulation functions of the pattern show large deviations [38]. Consequently, the reconstructed images of the particles from these holograms differ. Figure 8 illustrates a digital reconstruction by means of the fractional Fourier trans- 


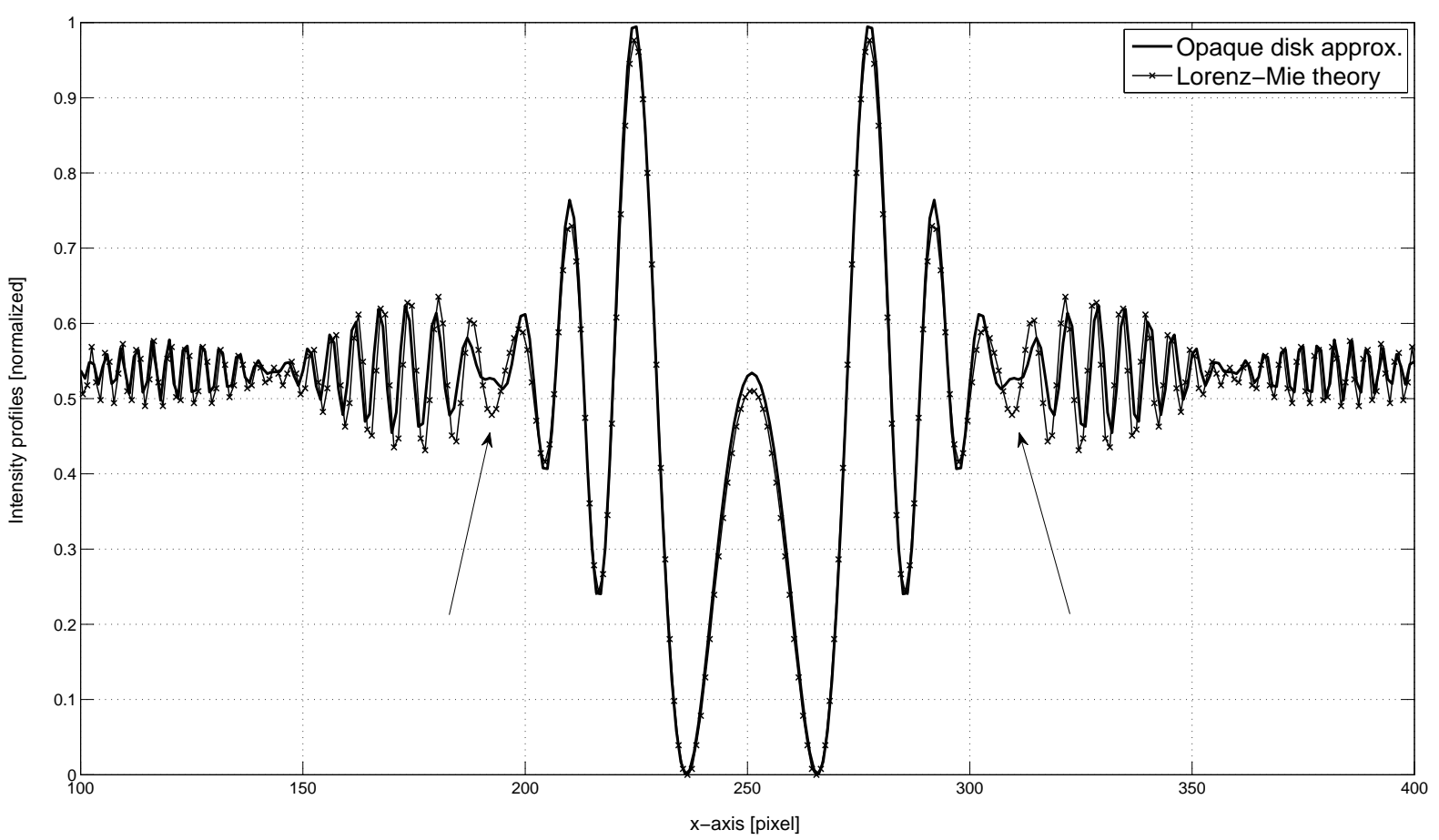

FIG. 7 Simulated intensity distribution in the plane of the CCD sensor with $\lambda=642 \mathrm{~nm}, n=1, n_{i}=1.33, D=20 \mu \mathrm{m}, z=3 \mathrm{~mm}$.

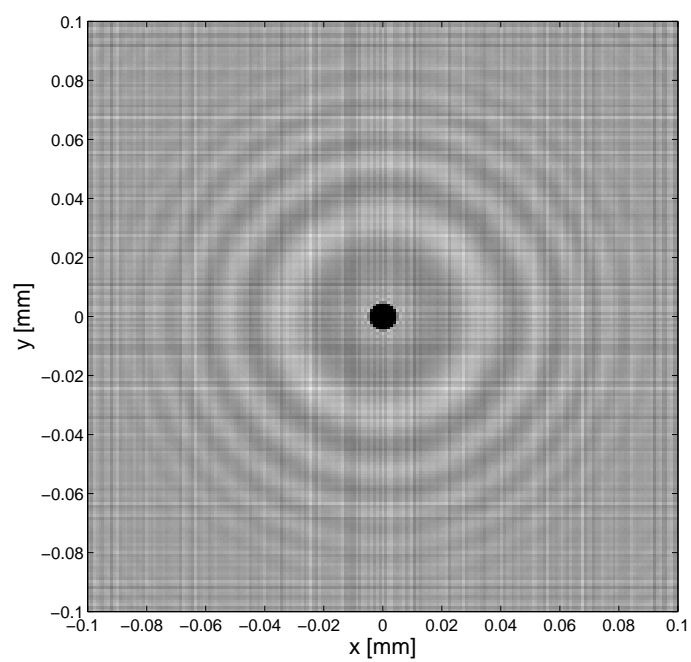

(a)

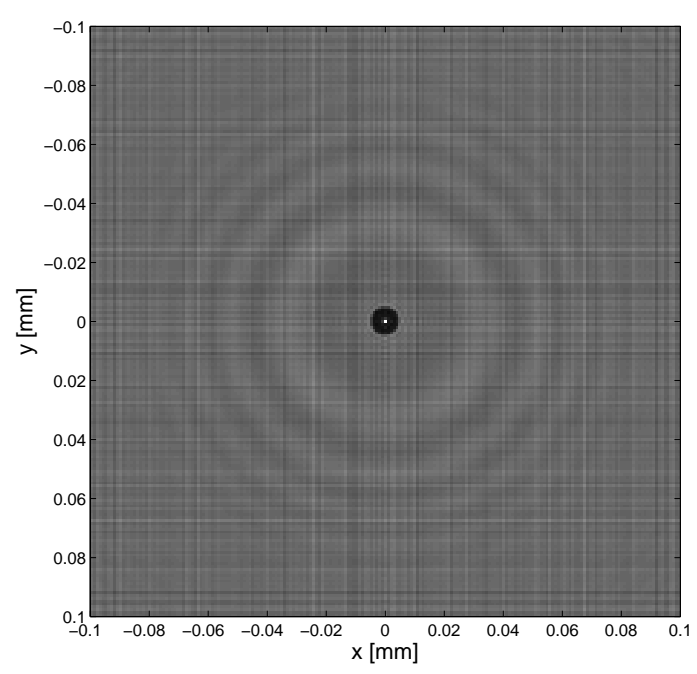

(b)

FIG. 8 Reconstruction by FRFT with $a_{\mathrm{xo}}=a_{y_{0}}=0.701$ in the cases of (a) the opaque disk approximation and (b) the near-field Lorenz-Mie scattering theory

formation. The optimal fractional orders from Eqs. (32) are $a_{x o}=a_{y o}=0.701$.

As one can see, in the case of the LMT in Figure 4(b), the centered light spot has been reconstructed whereas in the case of the opaque disk approximation, Figure 4(a), we obtained only the reconstructed image of a disk but not the centered light spot. Note that the spot of light at the center of the reconstructed image predicted by LMT been experimentally observed in [28]. To quantify the difference between these two results, the 2D-correlation coefficient of the Wigner distribution function of the profiles in Figure 7 has been computed [29]. This choice was largely motivated by the fact that in this phase space representation, the linear chirp is clearly identified (a pure linear chirp is a Dirac impulse in the phase space representation) and the aperture of the object, i.e., the diameter, is retained as a result. When the diameter is small, it is the linear chirp which is predominant, but, reciprocally, when the diameter is large, it is the aperture which is pre- dominant. The opaque disk approximation of a droplet gives us a good approximation, close to the LMT (which is the numerical standard), with a 2D-correlation coefficient equal to 0.96 . This comparison was carried out on the basis of a disk with a diameter of $20 \mu \mathrm{m}$, and located at a distance of $z=3 \mathrm{~mm}$ from the sensor. This implies a Fraunhofer coefficient (i.e., $\pi D^{2} /(2 \lambda z)$ ) equal to 0.326 . The study has been extended to Fraunhofer coefficients from 0.02 to 8.156, i.e., for $5 \mu \mathrm{m}<D<100 \mu \mathrm{m}$ at $\lambda=642 \mathrm{~nm}$. The opaque disk approximation of a droplet remains valid with a mean $2 \mathrm{D}$-correlation coefficient equal to 0.96 with a standard deviation of 0.02 . While this 2D-correlation coefficient is high, the absence of the light spot in the reconstructed image indicates that the modulation functions for these two cases do not agree. Therefore, we have to study another way of interpreting the holograms. 


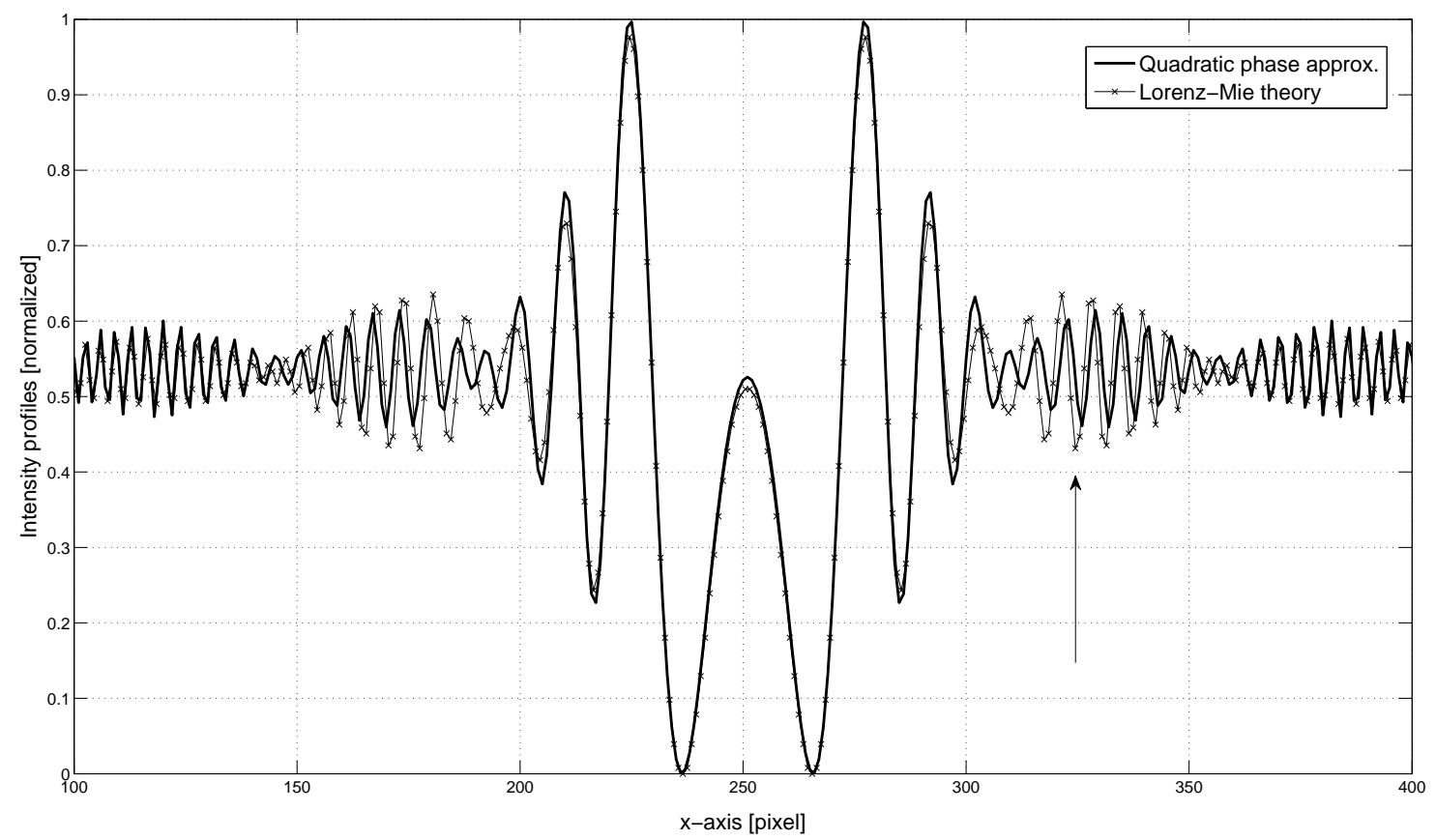

FIG. 9 Profiles of the simulated intensity distribution in the plane of the CCD sensor with $\lambda=642 \mathrm{~nm}, n=1, n_{i}=1.33, D=20 \mu \mathrm{m}, z=3 \mathrm{~mm}$.

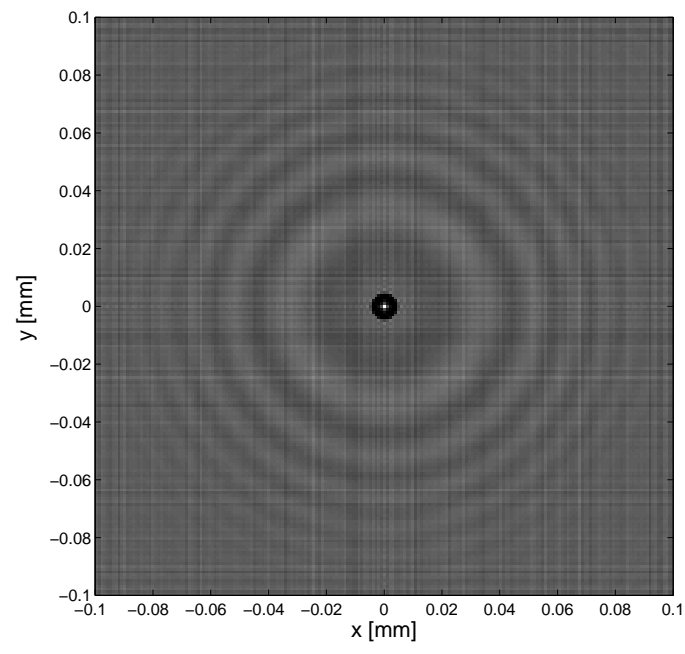

(a)

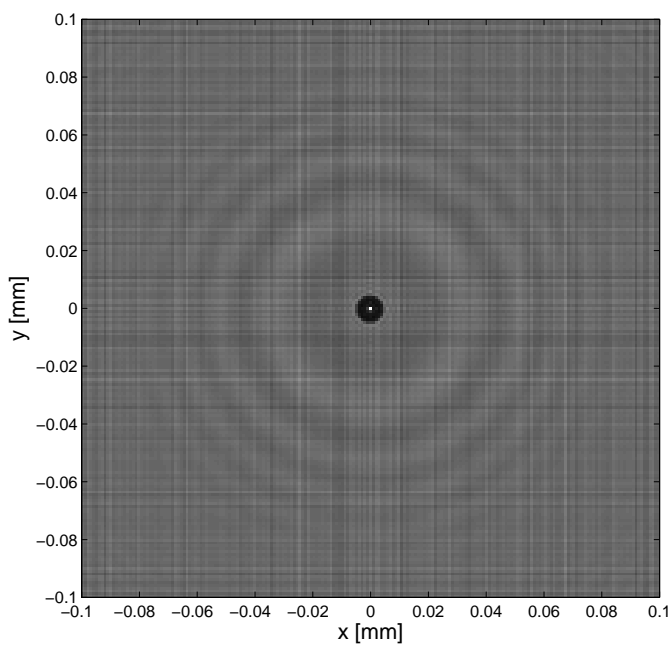

(b)

FIG. 10 Reconstruction by FRFT with $a_{x o}=a_{y 0}=0.701$ in the cases of (a) the quadratic phase approximation and (b) the near-field Lorenz-Mie scattering theory.

\subsection{The case of a droplet in the quadratic phase approximation}

Under the paraxial Gauss conditions, we assume that the droplet is equivalent to a thin lens. Then, the $p$ function of the droplet over the aperture $D$ can be described by

$$
p(s, \theta)=\left[1-\exp \left(-i \pi \kappa s^{2}\right)\right] \cdot Z_{0}^{0}(s, \theta),
$$

with $\kappa=D^{2} /(4 \lambda f)$ and $f=\frac{n_{i} \cdot D}{4\left(n_{i}-n\right)}$ the effective focal length of the ball-shaped lens. Note that $\kappa$ can be a complex number so as to take into account, for example, any absorption in the droplet. This point is not further addressed here. It is well known that

$$
\exp \left[-i \pi \kappa s^{2}\right] \cdot Z_{0}^{0}(s, \theta)=\sum_{\substack{n=0 \\ n \text { even }}}^{\infty} \gamma_{n}^{0}(\kappa) \cdot Z_{n}^{0}(s, \theta),
$$

where $n=0,2, \ldots$, and the Zernike moments $\gamma_{n}^{0}(\kappa)$ are given by

$$
\gamma_{n}^{0}=(n+1) \exp \left(-i \pi \frac{\kappa}{2}\right)(-i)^{\frac{n}{2}} \cdot j_{\frac{n}{2}}\left(\kappa \frac{\pi}{2}\right)
$$

Then, in the case of a circular system, i.e., $\delta=0$, the semianalytical expression for $G_{n}^{m}(\sigma, \varphi)$ in Eq. (23) is

$$
G_{n}^{0}(\sigma, \varphi)=2 \pi \sum_{\substack{n=0 \\ n \text { even }}}^{\infty} \gamma_{n}^{0} \cdot V_{n}^{0}(2 \pi \sigma, \chi),
$$

with $\gamma_{n}^{0}$ as defined in Eq. (37). Figure 9 illustrates the results of the quadratic approximation of the droplet compared to the LMT.

As one can see here, the two optical signals agree quite well. A minor shift of the linear chirp can be noticed. This is due to the modulation function's not being exactly the same as before. Nevertheless, in the reconstructions of these two holograms by a FRFT with $a_{x o}=a_{y o}=0.701$, illustrated in Figure 10), the light spot is visible in both cases.

Again, the quadratic phase approximation of a droplet gives us a good approximation, close to the numerical standard with a $2 \mathrm{D}$-correlation coefficient equal to 0.93 . Under the same 
conditions as the opaque disk approximations for Fraunhofer coefficients from 0.02 to 8.156 , the mean 2D-correlation coefficient reaches 0.93 with a standard deviation of 0.04 . Note that in the two previous approximations, the models of the droplet were defined using the zero and first order Maclaurin series of the phase function of the droplet (the paraxial approximation). In the next subsection, we propose to model the droplet with an approximation that we shall refer to as the quasi-spherical phase approximation; it is based on the idea of using a thickness function to extend the model beyond the paraxial approximation.

\subsection{The case of a droplet in the quasi-spherical phase approximation}

In this case, we must study Eqs. (10) and (12). The droplet is purely spherical-shaped, generally with a constant refractive index, denoted here $n_{i}$. The diameter of the droplet is again denoted $D$. In the theory of aberration, a pupil function can be considered as a plane or spherical wave. We need here an adequate description of the pupil function. Here, we adopt the same Zernike based approach as in subsection 4.1. Then, an appropriate transmittance function for the droplet will allow us to evaluate Eqs. (10) and (12). Consequently, the intensity distribution in the plane of the CCD sensor comes from a droplet whose transmittance function has been extended beyond the paraxial approximation. To do this, and by means of the developments introduced in [39], see Eqs. (5-1) and (5-2) on p. 74 , the total phase delay $\phi(\mathbf{r})$ of the wave at $\mathbf{r}=(\xi, \psi)$ when passing through the droplet equals

$$
\phi(\mathbf{r})=2 \pi\left(n_{i}-n\right) \frac{\Delta(\mathbf{r})}{\lambda},
$$

where $\Delta(\mathbf{r})$ is the thickness function of the deflected ray at $\mathbf{r}$. By recalling that $\mathbf{r}=\mathbf{s} D / 2$, we have $\widehat{\Delta}(\mathbf{s})=D \cdot\left(1-c^{2} s^{2}\right)^{1 / 2}$, with the numerical aperture $c=\left(n / n_{i}\right)$ such that $0<c<1$ and $\widehat{\Delta}(\mathbf{s})=\Delta(\mathbf{s} D / 2)$. It is important to note that in Eq. (39), we assumed that the coordinates of the entrance ray in the droplet and the exit ray are the same. In other words, the bending of the beam is not taken into account. Consequently, in this approximation, the phase transformation due to the droplet, which is nothing else than the pupil function $p$, can be written as

$$
p(s, \theta)=\left[1-\exp \left(i \pi \kappa_{n i} \sqrt{1-c^{2} \cdot s^{2}}\right)\right] \cdot Z_{0}^{0}(s, \theta),
$$

with $\kappa_{n i}=2\left(n_{i}-n\right) D / \lambda$. By considering [40], the pupil function in Eq. (40) can be expanded in terms of circle polynomials:

$$
\begin{gathered}
\exp \left(i \pi \kappa_{n i} \sqrt{1-c^{2} \cdot s^{2}}\right) Z_{0}^{0}(s, \theta)= \\
\sum_{\substack{n=0 \\
n \text { even }}}^{\infty} \gamma_{n}^{0}\left(-\pi \kappa_{n i} \cdot u_{c}\right) \cdot Z_{n}^{0}(s, \theta)
\end{gathered}
$$

with $u_{c}=1-\sqrt{1-c^{2}}$ and

$$
\begin{aligned}
\gamma_{n}^{0}(x) & =(n+1) \cdot\left[\frac{x}{2} \cdot \mathbf{j}_{\frac{n}{2}-1} \cdot \mathbf{h}_{\frac{n}{2}}^{(2)}-\frac{x}{2 v_{c}} \mathbf{j}_{\frac{n}{2}} \cdot \mathbf{h}_{\frac{n}{2}+1}^{(2)}\right], \\
v_{c} & =\frac{1-\sqrt{1-c^{2}}}{1+\sqrt{1-c^{2}}}
\end{aligned}
$$

The $\mathbf{j}_{n}$ are the spherical Bessel functions of the first kind and $\mathbf{h}_{n}^{(2)}$ are the spherical Hankel functions of the second kind. Each spherical Bessel function has argument $x / 2$ and each spherical Hankel function has argument $x /\left(2 v_{c}\right)$. Then, in the case of a circular system, i.e., $\delta=0$, the semi-analytical expression for $G_{n}^{m}(\sigma, \varphi)$ in Eq. (23) is

$$
G_{n}^{0}(\sigma, \varphi)=2 \pi \sum_{\substack{n=0 \\ n \text { even }}}^{\infty} \gamma_{n}^{0} \cdot V_{n}^{0}(2 \pi \sigma, \chi),
$$

with $\gamma_{n}^{0}$ defined in Eq. (42).

The result is illustrated in Figure 11 and compared to the LMT simulation. The quasi-spherical phase approximation of a droplet is close to the numerical standard with the presence of the light spot at the center. For the Fraunhofer coefficients from 0.02 to 8.156 , the mean 2D-correlation coefficient reaches 0.95 with a standard deviation of 0.03 . As a result, the description of the droplet as a thickness function seems to yield a satisfactory approximation. Again here, the light spot is reconstructed from these two holograms by a FRFT with $a_{x o}=a_{y o}=0.701$, see Figure 12. Finally, the profile of the three reconstructions shown in Figure 13). Qualitatively, the results obtained through approximations are close to the numerical standard LMT.

At the moment, there is only a qualitative and not quantitative comparison. Further study will permit a quantitative comparison. In this publication we limit ourselves to the first estimate. However, the other advantage of this point of view, i.e. the thickness function, is that it is probably possible to consider an other shape of the object, provided that a circular symmetrical exists.

\section{NUMERICAL ESTIMATION OF THE ZERNIKE COEFFICIENTS}

Recall that $G_{n}^{m}$ in Eq. (23) allows us to describe the object function in the sense of holography, from a general point of view, and its knowledge is important when a metrology for the particle must be realized, for example, to measure its diameter. In Eq. (23), the characteristic function of the particle is contained in the Zernike coefficients. In the previous cases, the Zernike coefficients were clearly defined from the shape of the particle (alternatively, of the inclusion). However, there may be random physical situations where the pure spherical shape of the particle may be disturbed. Then, the expression of the pupil function in Eq. (10) becomes a complicated mathematical expression, for example if the phase $\Phi(s, \theta)$ of the pupil function contains non-linear chirps (i.e., aberrations). In this case, the Zernike coefficients do not have a closed form. Consequently, the Zernike coefficients in Eq. (12) should be numerically evaluated. A good digital evaluation of the Zernike coefficients in Eq. (12) means that it is possible from the estimated Zernike coefficients to reconstruct the initial function, that may contain high-order spatial frequencies. For this, the double exponential formula (DE) of numerical integration is used [45]. The aim of this formula is to find the optimal change of variable which transforms the original integral over a finite interval to an integral over an infinite interval. Such transfor- 


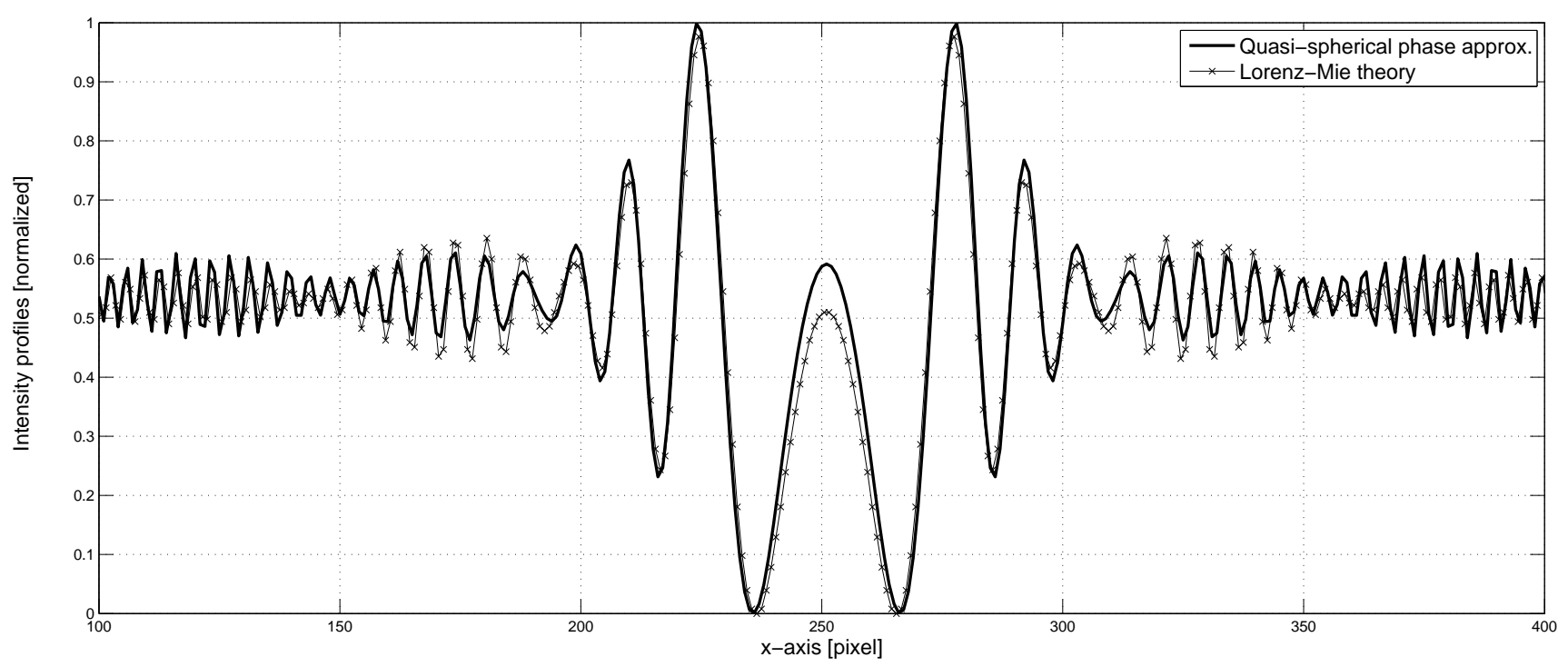

FIG. 11 Profiles of the simulated intensity distribution in the plane of the CCD sensor with $\lambda=642 \mathrm{~nm}, n=1, n_{i}=1.33, D=20 \mu \mathrm{m}, z=3 \mathrm{~mm}$.

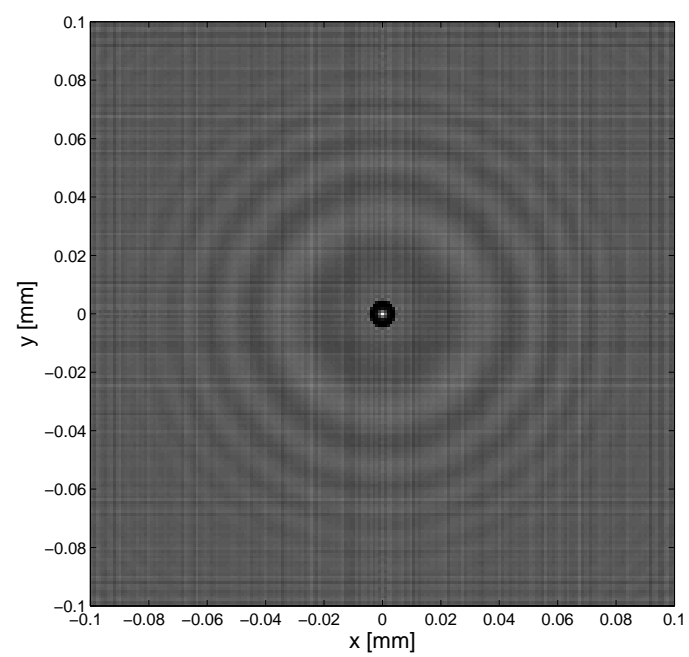

(a)

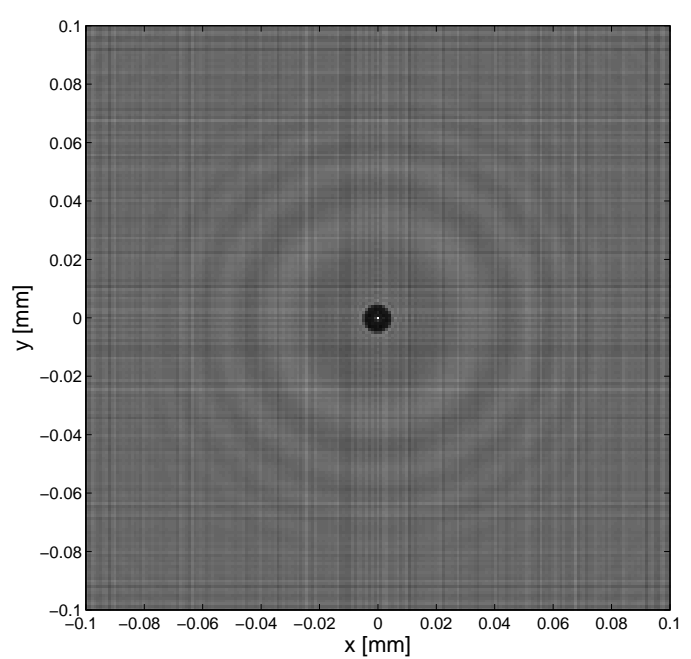

(b)

FIG. 12 Reconstruction of the image of the droplet in the cases of (a) the quasi-spherical phase approximation and (b) the near-field Lorenz-Mie scattering theory.

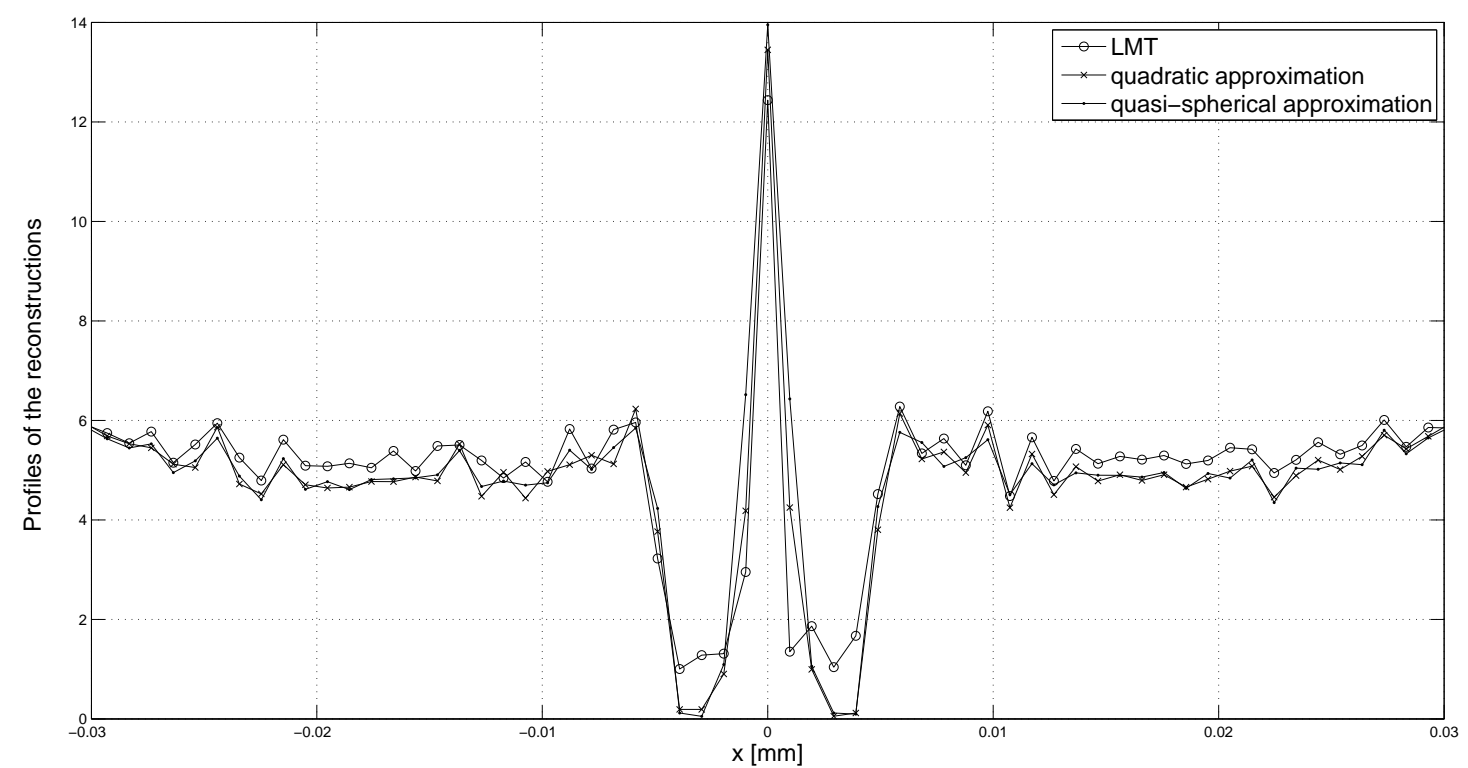

FIG. 13 Profiles of the reconstruction of the image of the droplet in the three cases: LMT, quadratic approximation, and quasi-spherical phase approximation. 
mations of the variables allow applying the trapezoidal rule over $(-\infty,+\infty)$. For Eq. (12), the change of variables is

$$
\begin{aligned}
& s=\phi(u)=\frac{1}{2}\left[1+\tanh \left(\frac{\pi}{2} \sinh (u)\right)\right], \\
& \theta=\psi(v)=\pi\left[1+\tanh \left(\frac{\pi}{2} \sinh (v)\right)\right],
\end{aligned}
$$

where

$$
\phi(-\infty)=0, \quad \phi(\infty)=1, \quad \psi(-\infty)=0, \quad \psi(\infty)=2 \pi .
$$

In this way, the integral expression for the Zernike coefficient, denoted here by $\Gamma_{n}^{m}$, becomes

$$
\begin{aligned}
& \Gamma_{n}^{m}= \\
& \quad \frac{(n+1)}{\pi} \cdot \int_{\mathbb{R}^{2}} F_{n}^{m}(\phi(u), \psi(v)) \cdot \phi(u) \phi^{\prime}(u) \psi^{\prime}(v) d u d v .
\end{aligned}
$$

with

$$
F_{n}^{m}(s, \theta)=A(s, \theta) \cdot \exp [i \Phi(s, \theta)] \cdot \overline{Z_{n}^{m}(s, \theta)} .
$$

The amplitude $A(s, \theta)$ can be a constant or a Gaussian function, denoted by $\exp \left(-\alpha s^{2}\right), \alpha \in \mathbb{R}^{+}$. The phase $\Phi(s, \theta)$ is a real function of $s$ and $\theta$. Note that

$$
\begin{aligned}
\phi^{\prime}(u) & =\frac{\pi}{4} \frac{\cosh (u)}{\cosh ^{2}\left(\frac{\pi}{2} \sinh (u)\right)} \\
& =O\left(\exp \left(-\frac{\pi}{2}(1-\alpha) \exp |u|\right)\right), \quad|u| \longrightarrow \infty,
\end{aligned}
$$

and

$$
\begin{aligned}
\psi^{\prime}(v) & =\frac{\pi^{2}}{2} \frac{\cosh (v)}{\cosh ^{2}\left(\frac{\pi}{2} \sinh (v)\right)} \\
& =O\left(\exp \left(-\frac{\pi}{4}(1-\alpha) \exp |v|\right)\right), \quad|v| \longrightarrow \infty
\end{aligned}
$$

holds for arbitrary $0<\alpha<1$ [46]. The integrand decays doubly exponentially after the transformation. This is the reason it is called the double exponential formula. After this transformation, the integrand in Eq. (46) has singular points in the complex plane where there are zeros $z_{0}$ of $\phi^{\prime}(u)$, which are given by

$$
\cosh \left(z_{o}\right)=0, \quad z_{o}= \pm i \frac{\pi}{2}+i 2 \pi \mathbb{Z},
$$

and the poles $p_{o}$ are given by

$$
\begin{gathered}
\cosh ^{2}\left(\frac{\pi}{2} \sinh \left(p_{o}\right)\right)=0 \\
p_{o}= \pm i \arcsin (1)= \pm i \frac{\pi}{2}+i 2 \pi \mathbb{Z} .
\end{gathered}
$$

Then the integrand in Eq. (46) is regular in the strip [47]

$$
|\Im\{u\}|<d=\frac{\pi}{2}
$$

where $d$ is the minimum distance between the singular points of the integrand in Eq. (46) and the real axis. In order to evaluate the integral in Eq. (46), we employ the trapezoidal formula

$$
\begin{aligned}
\Gamma_{n}^{m}= & \frac{(n+1)}{\pi} \cdot \delta_{u} \delta_{v} \sum_{k=-N}^{+N} \sum_{l=-N}^{+N} F_{n}^{m}\left[\phi\left(k \delta_{u}\right), \psi\left(l \delta_{v}\right)\right] \\
& \phi\left(k \delta_{u}\right) \phi^{\prime}\left(k \delta_{u}\right) \psi^{\prime}\left(l \delta_{v}\right)+\Delta \gamma_{\delta}
\end{aligned}
$$

with $\delta_{u, v}$ the constant sampling period along the $u$-axis and the $v$-axis, and $\Delta \gamma_{\delta}$ is linked to the discretization and the truncation errors given in [47], Eqs. (3.13) and (3.19), pp. 909910 by

$$
\begin{aligned}
\Delta \gamma_{\delta}= & O\left(\exp \left[-\frac{2 \pi d}{\delta_{u, v}}\right]\right) \\
& +O\left(\frac{\pi}{4} \exp \left(-\frac{\pi}{2}(1-\alpha) \exp \left(N \delta_{u, v}\right)\right)\right) .
\end{aligned}
$$

Next, the sampling distance $\delta_{u, v}$ is chosen such that the truncation error and discretization error have the same order of magnitude. Thus, setting

$$
\exp \left[-\frac{2 \pi d}{\delta_{u, v}}\right]=\frac{\pi}{4} \exp \left[-\frac{\pi}{2}(1-\alpha) \exp \left(N \delta_{u, v}\right)\right],
$$

we can determine the sampling period $\delta_{u, v}$ or the number of evaluation $N$. To illustrate our purpose, the results of Eqs. (37) and (53) are compared in Figure 14 . In this case, the $\kappa$ coefficient is equal to 7.7296 .

The comparison produces an error, which is denoted here by $\Delta_{e}$, and is defined by

$$
\Delta_{e}=\max \left(|| \Re\left(\Gamma_{n}^{m}\right)-\Re\left(\gamma_{n}^{m}\right)|+| \Im\left(\Gamma_{n}^{m}\right)-\Im\left(\gamma_{n}^{m}\right)||\right) .
$$

In the case presented in Figure 14, with $m=0$, the maximal error is $0.557 \cdot 10^{-13}$ over the range $n=[0,34]$. Secondly, the comparison between Eqs. (42) and (53) is illustrated in Figure 15).

The maximal error is equal to $0.519 \cdot 10^{-13}$ over the same range. This formula seems to work well for a pure-phase pupil function containing high order aberrations. For example, if $p$ is defined by

$$
p(s, \theta)=\exp \left(i \alpha R_{3}^{1}(s) \cos \theta\right) \simeq \sum_{n, m} \Gamma_{n}^{m} \cdot Z_{n}^{m}(s, \theta), \alpha=1,
$$

the numerical estimation of the Zernike coefficients $\Gamma_{n}^{m}$ can be compared to the theoretical Zernike coefficients given in [48], Eq. (A18). To do this, Figure 16 present the numerical and theoretical Zernike coefficients, with real and imaginary parts denoted here by $\Re$ and $\Im$. The maximum error from Eq. (56) is evaluated at $0.854 \cdot 10^{-15}$ over the range $(n, m)=[0,20] \times[-20,20] . \mathrm{f}$

\section{CONCLUSIONS}

In this paper, we have proposed a mathematical model of the intensity distribution for a particle's hologram. Three approximations of the pupil function of a spherical particle have been considered: the opaque disk, the quadratic phase approximation, and the quasi-spherical phase approximation. We have compared these three approximations with the nearfield Lorenz-Mie scattering theory. The quadratic phase and quasi-spherical phase approximations of the pupil function have been represented in terms of the circle polynomials. These three points of view are rather good approximations but as we have seen, the opaque particle approximation fails to explain the presence of a light spot at the center of the reconstructed images unlike the other two approximation. The 


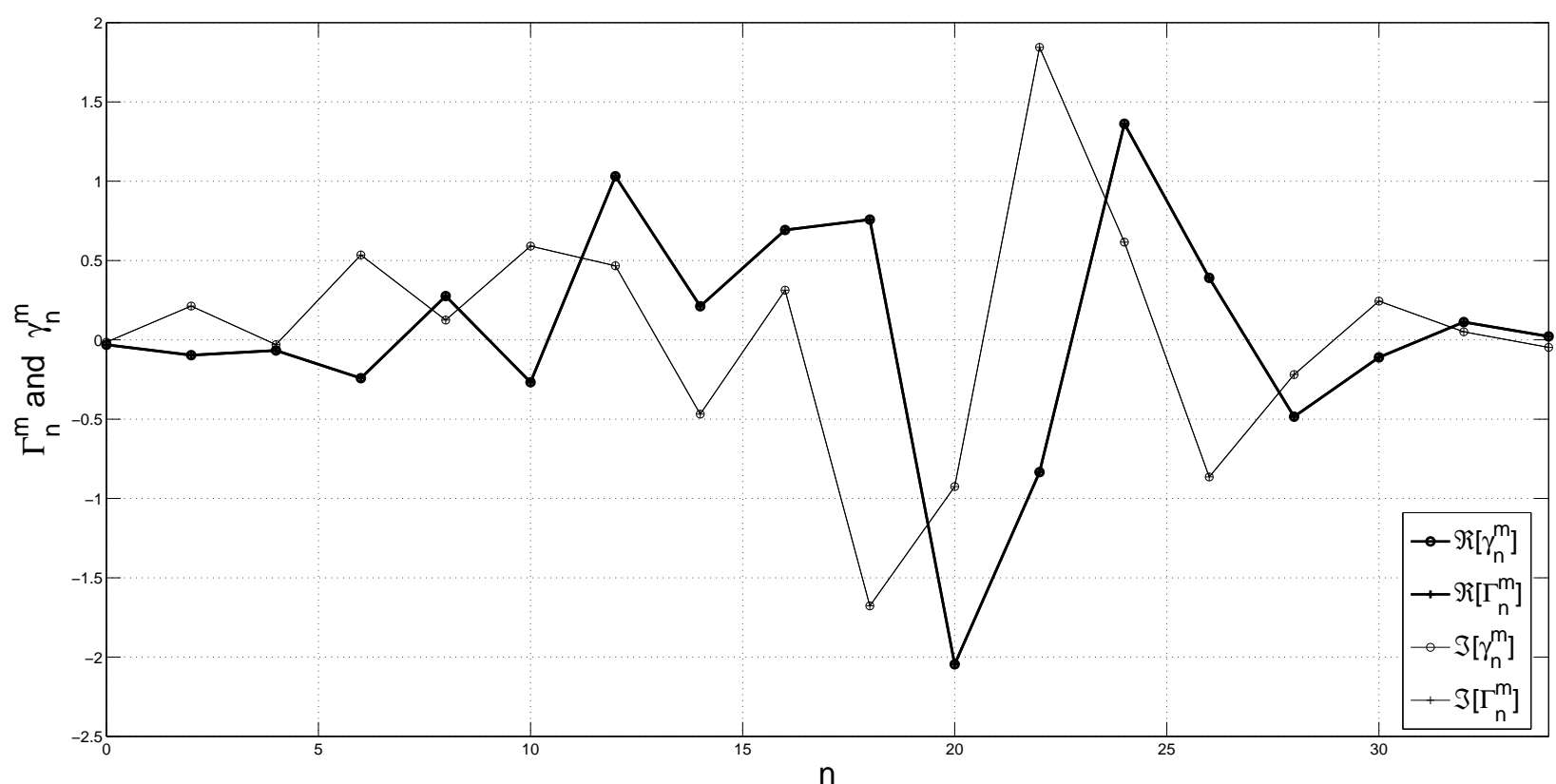

FIG. 14 Comparison between the theoretical $\gamma_{n}^{m}$ and numerically obtained $\Gamma_{n}^{m}$ Zernike coefficients with $m=0$ in the case of a droplet described by a quadratic phase approximation. $N=100, \delta_{u}=\delta_{v}=0.0486167$.

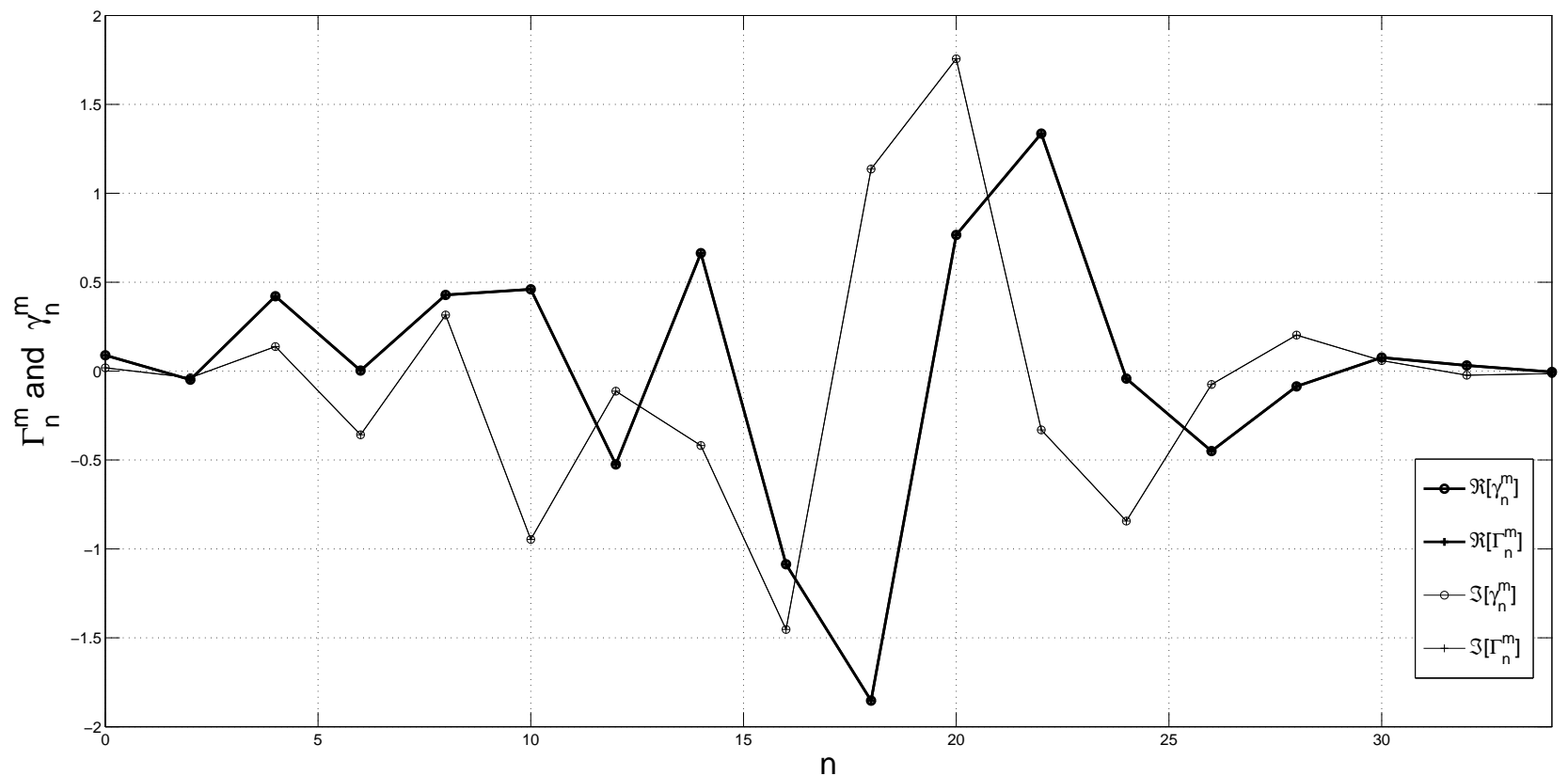

FIG. 15 Comparison between the theoretical $\gamma_{n}^{m}$ and numerically obtained $\Gamma_{n}^{m}$ Zernike coefficients with $m=0$ in the case of a droplet in the quasi-spherical phase approximation. $N=100, \delta_{u}=\delta_{v}=0.0486167$.

quasi-spherical approximation is considerably better than the quadratic approximation because it takes into account the true thickness function. The main simplifying approximation in our analysis has been the beam has the same coordinates at its entrance into and its exit from the droplet. The limits of our models are included within the Fresnel's approximation, since the models are developed using the Fresnel's integral. However, we observe that here the diameters increased beyond $100 \mu \mathrm{m}$, the model of the quadratic phase approximation was worse than the model of the quasi-spherical approximation, of course, in the experimental context of this publication. Note again that the limitation is the object's refractive index should be higher than the refractive index of water (or surrounding medium). Next, if the thickness function is not a trivial function, the theoretical expression for the Zernike coefficients does not have a closed form. Evaluating the Zernike coefficients is then not straightforward and a satisfactory evaluation of the intensity distribution in the hologram depends on the quality of the numerical estimation of the Zernike coefficients. Consequently, a numerical evaluation is necessary and we have adopted the double exponential formula, which is known for its high accuracy. This formula works well even for pupil functions containing high order aberrations.

\section{ACKNOWLEDGEMENTS}

This work was partially supported by the French National Agency under grant ANR-2011-NANO-008 "NANOMORPH". The authors acknowledge the partial financial support of the French Agence Nationale de la Recherche (ANR), through the program Investissements 

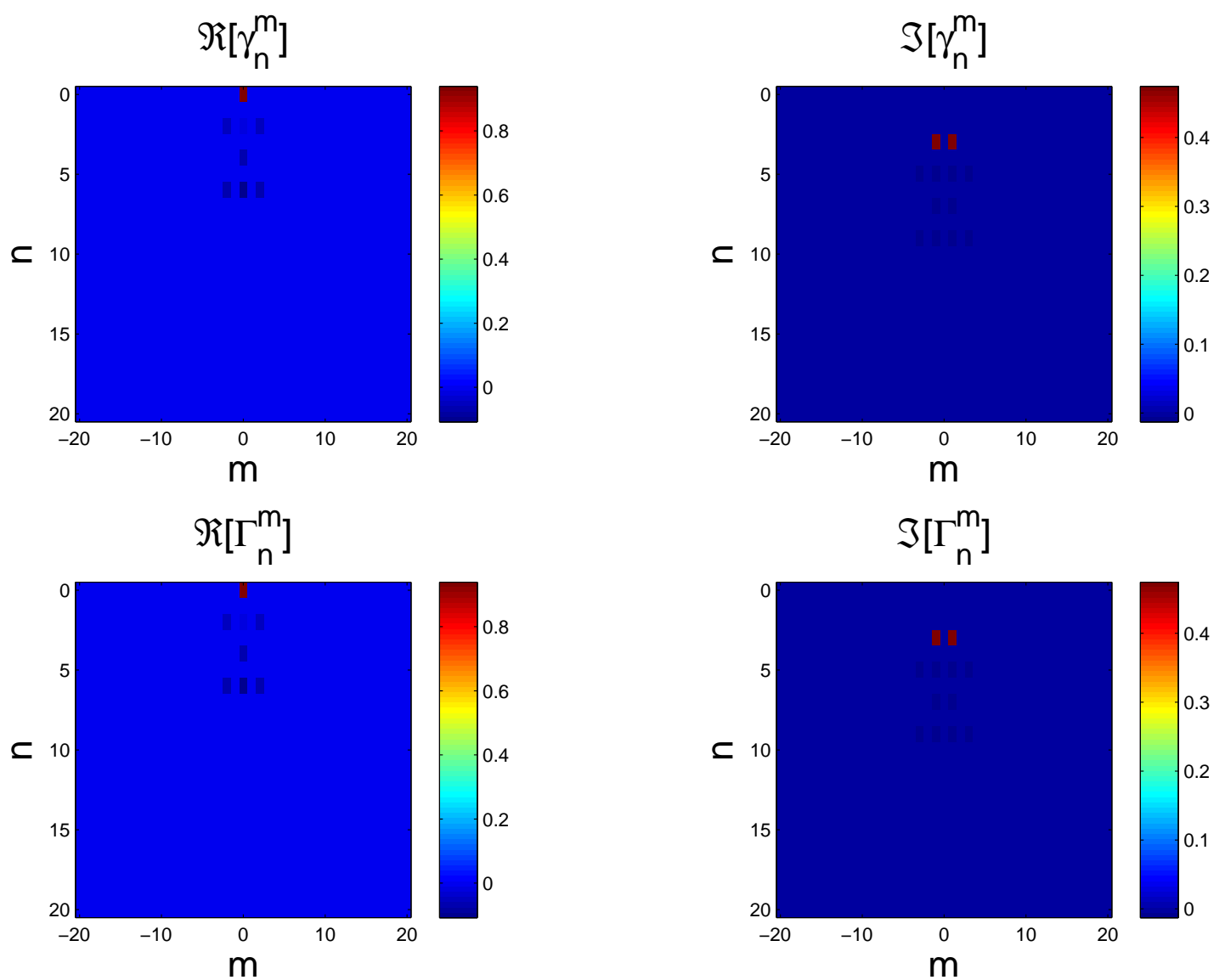

FIG. 16 Comparison of the theoretical $\gamma_{n}^{m}$ and the numerically obtained $\Gamma_{n}^{m}$ Zernike coefficients for the coma function of the pupil. $N=100, \delta_{u}=\delta_{v}=0.0486167$.

d'Avenir (ANR-10-LABEX-09-01). This work was partially supported by the LABEX EMC3 (Energy Materials and Clean Combustion Center) of the Université de Rouen, within the program "3D" operated by the French National Research Agency (ANR).

\section{A THE MATRICES $\mathbb{M}_{i}$ AND $\mathbb{M}_{t}$}

Here the incident matrix is defined by means of the experimental values given in Figure 2 . Then, along the $x$-axis, the incident matrix is defined by

$$
\begin{gathered}
\mathbb{M}_{i(x, y)}=\left(\begin{array}{ll}
1 & \frac{\delta}{n} \\
0 & 1
\end{array}\right)\left(\begin{array}{cc}
1 & 0 \\
\frac{n_{a}-n}{d / 2} & 1
\end{array}\right)\left(\begin{array}{cc}
1 & \frac{e_{3}}{n_{a}} \\
0 & 1
\end{array}\right) \\
\cdot\left(\begin{array}{cc}
1 & 0 \\
-\frac{1}{f_{2}} & 1
\end{array}\right)\left(\begin{array}{cc}
1 & \frac{e_{2}}{n_{a}} \\
0 & 1
\end{array}\right)\left(\begin{array}{cc}
1 & 0 \\
-\frac{1}{f_{1}} & 1
\end{array}\right)\left(\begin{array}{cc}
1 & \frac{e_{1}}{n_{a}} \\
0 & 1
\end{array}\right)
\end{gathered}
$$

and along the $y$-axis, the incident matrix is the same when the optical setup is symmetric. To obtain an astigmatic optical system, it is possible to modify one of the focal lengths. Now, for the transmitted matrix, we have

$$
\mathbb{M}_{t(x, y)}=\left(\begin{array}{cc}
1 & \frac{z}{n_{a}} \\
0 & 1
\end{array}\right)\left(\begin{array}{cc}
1 & 0 \\
\frac{n-n_{a}}{-d / 2} & 1
\end{array}\right)\left(\begin{array}{cc}
1 & \frac{d-\delta}{n} \\
0 & 1
\end{array}\right)
$$

\section{B THEORETICAL DEVELOPMENT OF $G_{n}^{m}$}

To get a semi-analytical computation method for the remaining integral in Eq. (20), the approach is as follows. Firstly, write

$$
\begin{aligned}
\exp \left[i \mathbf{s}^{T} \mathbb{L}_{t} \mathbf{s}\right]=\exp [ & i \frac{1}{2} s^{2}\left(\mathbb{L}_{t(1,1)}+\mathbb{L}_{t(2,2)}\right) \\
& \left.+i \frac{1}{2} s^{2}\left(\mathbb{L}_{t(1,1)}-\mathbb{L}_{t(2,2)}\right) \cos (2 \theta)\right]
\end{aligned}
$$

Then expand, using $\beta$-coefficients as before,

$$
\begin{aligned}
\exp \left[i \frac{1}{2} s^{2}\left(\mathbb{L}_{t(1,1)}-\mathbb{L}_{t(2,2)}\right) \cos (2 \theta)\right] \\
=\sum_{q=-\infty}^{+\infty} \sum_{p=0}^{\infty} i^{q} \cdot \beta_{|2 q|+2 p}^{|2 q|}(\delta) \cdot Z_{|2 q|+2 p}^{2 q}(s, \theta)
\end{aligned}
$$

with $\delta=\frac{1}{2}\left(\mathbb{L}_{t(1,1)}-\mathbb{L}_{t(2,2)}\right)$ and where the expansion coefficients $\beta_{|2 q|+2 p}^{|2 q|}$ are expressed explicitly in terms of the hypergeometric functions ${ }_{2} F_{3}$, cf. [26], as

$$
\begin{aligned}
& \beta_{|2 q|+2 p}^{|2 q|}(\delta)=d_{0}^{0}(-1)^{r}(2|2 q|+4 r+1)\left(\frac{1}{2} \delta\right)^{|2 q|+2 r} \\
& \cdot{ }_{2} F_{3}\left(\begin{array}{ccc}
r+\frac{1}{2} & |2 q|+r+\frac{1}{2} & \\
\frac{1}{2} & |2 q|+2 r+\frac{3}{2} & |2 q|+2 r+1
\end{array} ;-\frac{1}{4} \delta^{2}\right)
\end{aligned}
$$

in the case where $2 r-p=0$ and

$$
\begin{aligned}
& \beta_{|2 q|+2 p}^{|2 q|}(\delta)=d_{0}^{1}(-1)^{r}(2|2 q|+4 r-1)\left(\frac{1}{2} \delta\right)^{|2 q|+2 r} \\
& \cdot{ }_{2} F_{3}\left(\begin{array}{ccc}
r+\frac{1}{2} & |2 q|+r+\frac{1}{2} & \\
\frac{3}{2} & |2 q|+2 r+1 & |2 q|+2 r+\frac{1}{2}
\end{array} ;-\frac{1}{4} \delta^{2}\right)
\end{aligned}
$$


in the case where $2 r-p=1$. In Eqs. (62) and (63), the coefficients $d_{0}^{0}$ and $d_{0}^{1}$ are defined as follows:

$$
\begin{aligned}
& d_{0}^{0}=\frac{(2 r) !(2|2 q|+2 r) !}{r !(|2 q|+r) !(2|2 q|+4 r+1) !}, \\
& d_{0}^{1}=\frac{(2 r) !(2|2 q|+2 r) !}{r !(|2 q|+r) !(2|2 q|+4 r) !} .
\end{aligned}
$$

When Eq. (61) is introduced into Eq. (21), we see that there arises the product of two circle polynomials,

$$
Z_{n}^{m}(s, \theta) \cdot Z_{|2 q|+2 p}^{2 q}(s, \theta) .
$$

In [23], Eqs. (40)-(44) these products are linearized:

$$
Z_{n}^{m}(s, \theta) \cdot Z_{|2 q|+2 p}^{2 q}(s, \theta)=\sum_{t} A_{n,|2 q|+2 p, t}^{m, 2 q, m+2 q} \cdot Z_{t}^{m+2 q}(s, \theta)
$$

The summation range over $t$ is

$$
t=\max (|m+2 q|,|m-| 2 q|-2 p|)(2)(n+|2 q|+2 p)
$$

where $\mathrm{a}(2) \mathrm{b}$ denotes $a, a+2, \ldots, b$ when $b-a$ is non-negative and even. Then, $A$ can be given in terms of Wigner or ClebschGordan coefficients [44, 43]:

$$
A_{j, l, n}^{i, k, m}=\left|C_{\frac{i}{2}, \frac{k}{2}, \frac{l}{2}, \frac{n}{2}}^{2}\right|^{2}
$$

Note that when $t$ is not as in Eq. (24), then $A_{j, l, n}^{i, k, m}=0$. The integral in Eq. (21) is now

$$
\begin{aligned}
\int_{0}^{1} \int_{0}^{2 \pi} & \exp \left[i \chi s^{2}\right] Z_{t}^{m+2 q}(s, \theta) \\
& \cdot \exp [-i 2 \pi \sigma s \cos (\varphi-\theta)] s d s d \theta,
\end{aligned}
$$

with $\chi=\frac{1}{2} \cdot \operatorname{Tr}\left(\mathbb{L}_{t}\right)$. This latter integral can be expressed in term of $V$ functions from the extended Nijboer-Zernike theory $[26,24]$. Indeed, we have

$$
\begin{aligned}
\int_{0}^{1} \int_{0}^{2 \pi} e^{i \chi s^{2}} \cdot Z_{t}^{m+2 q}(s, \theta) \cdot e^{-i 2 \pi \sigma s \cos (\varphi-\theta)} s d s d \theta \\
=2 \pi(i)^{m+2 q} \cdot V_{t}^{|m+2 q|}(2 \pi \sigma, \chi) \cdot e^{i(m+2 q) \varphi},
\end{aligned}
$$

and the power-Bessel series for $V$ is given by Eqs. (26) and (27). This yields the final result of the semi-analytical formula for the integral $G_{n}^{m}$ that we were looking for:

$$
\begin{aligned}
G_{n}^{m}\left(\sigma^{\prime}, \vartheta\right)=2 & \pi \sum_{q=-\infty}^{+\infty} \sum_{n, m, p}^{\infty} \sum_{t} \gamma_{n}^{m} \cdot(-i)^{m+q} \cdot A_{n,|2 q|+2 p, t}^{m, 2 q, m+2 q} \\
\cdot & \beta_{|2 q|+2 p}^{|2 q|}(\delta) \cdot V_{t}^{|m+2 q|}(2 \pi \sigma, \chi) \cdot e^{i(m+2 q) \varphi} .
\end{aligned}
$$

\section{References}

[1] S. Coëtmellec, N. Verrier, M. Brunel, and D. Lebrun, "General formulation of digital in-line holography from correlation with a chirplet function," J. Europ. Opt. Soc. Rap. Public. 5, 10027 (2010).

[2] M. Brunel, H. Shen, S. Coëtmellec, D. Lebrun, and K. Aït Ameur, "Femtosecond digital in-line holography with the fractional Fourier transform: application to phase-contrast metrology," Appl. Phys. B-Lasers 0. 106, 583-591 (2012).

[3] M. Brunel, H. Shen, S. Coëtmellec, D. Lebrun, and K. Aït Ameur, "Phase contrast metrology using digital in-line holography: general models and reconstruction of phase discontinuities," J. Quant. Spectrosc. Ra. 126, 113-121 (2012).
[4] H. Shen, S. Coëtmellec, and M. Brunel, "Simultaneous 3D location and size measurement of spherical bubbles using cylindrical interferometric out-of-focus imaging," J. Quant. Spectrosc. Ra. 131, 153-159 (2012).

[5] Y. Gu, and G. Gbur, "Scintillation of Airy beam arrays in atmospheric turbulence," Opt. Lett. 35, 3456-3458 (2010).

[6] L. Wind, L. Hofer, A. Nagy, P. Winkler, A. Vrtala, and W. Szymanski, "Light scattering from droplets with inclusions and the impact on optical measurement of aerosols," J. Aerosol Sci. 35, 1173-1188 (2004).

[7] A. Quérel, P. Lemaitre, and E. Porcheron, "Caractérisation des écoulements d'air produits lors de la chute d'une goutte de pluie," in Proceedings to the $13^{\text {th }}$ Congrès Francophone de Techniques Laser, 193-201 (CORIA, Rouen, 2012) in French.

[8] J. Wang, G. Gouesbet, G. Gréhan, Y. Han, and S. Saengkaew, "Morphology-dependent resonances in an eccentrically layered sphere illuminated by a tightly focused off-axis Gaussian beam: parallel and perpendicular beam incidence," J. 0pt. Soc. Am. 28, 1849-1859 (2011).

[9] K. Y. Lai, N. Dayan, and M. Kerker, "Scavenging of aerosol particles by a falling water drop," J. Atmos. Sci. 35, 674-682 (1978).

[10] S. A. Collins, "Lens-system diffraction integral written in terms of matrix optics," J. Opt. Soc. Am. 60, 1168-1177 (1970).

[11] T. Alieva, and M. J. Bastiaans, "Properties of the linear canonical integral transformation," J. Opt. Soc. Am. A 24, 3658-3665 (2007).

[12] R. K. Luneburg, Mathematical Theory of Optics (University of California Press, Berkeley, 1966).

[13] H. M. Ozaktas, Z. Zalevsky, and M. Alper Kutay, The Fractional Fourier Transform: with Applications in Optics and Signal Processing (Wiley, Chicester, 2001).

[14] Y. Cai, and Q. Lin, "Propagation of elliptical Gaussian beam through misaligned optical systems in spatial domain and spatialfrequency domain," Opt. Laser Technol. 34, 415-421 (2002).

[15] N. Verrier, C. Remacha, M. Brunel, D. Lebrun, and S. Coëtmellec, "Micropipe flow visualization using digital in-line holographic microscopy," Opt. Express 18, 7807-7819 (2010).

[16] N. Verrier, S. Coëtmellec, M. Brunel, D. Lebrun, and A. J. E. M. Janssen, "Digital in-line holography with an elliptical, astigmatic Gaussian beam: wide-angle reconstruction," J. 0pt. Soc. Am. A 25, 1459-1466 (2008).

[17] C. Zheng, D. Zhao, and X. Du, "Analytical expression of elliptical Gaussian beams through nonsymmetric systems with an elliptical aperture," Optik 117, 296-298 (2005).

[18] J. J. Wen, and M. A. Breazeale, "A diffraction beam expressed as the superposition of Gaussian beams," J. Acoust. Soc. Am. 83, 1752-1756 (1988).

[19] X. Du, and D. Zhao, "Propagation of decentered elliptical Gaussian beams in apertured and nonsymmetrical optical systems," J. Opt. Soc. Am. A 23, 625-631 (2006).

[20] X. Du, and D. Zhao, "Propagation of elliptical Gaussian beams in apertured and misaligned optical systems," J. Opt. Soc. Am. A 23, 1946-1950 (2006).

[21] X. Du, and D. Zhao, "Propagation of elliptical Gaussian beams modulated by an elliptical annular aperture," J. Opt. Soc. Am. A 24, 444-450 (2007).

[22] A. J. E. M. Janssen, "New analytic results for the Zernike circle polynomials from a basic result in the Nijboer-Zernike diffraction theory," J. Europ. Opt. Soc. Rap. Public. 6, 11028 (2011). 
[23] A. J. E. M. Janssen, "Computation of Hopkins' 3-circle integrals using Zernike expansions," J. Eur. Opt. Soc. Rapid. Public. 6, 11059 (2011).

[24] A. J. E. M. Janssen, "Extended Nijboer-Zernike approach for the computation of optical point-spread functions," J. Opt. Soc. Am. A 19, 849-857 (2002).

[25] S. van Haver, and A. J. E. M. Janssen, "Advanced analytic treatment and efficient computation of the diffraction integrals in the extended Nijboer-Zernike theory," J. Eur. Opt. Soc. Rapid. Public. 8, 13044 (2013).

[26] F. Nicolas, S. Coëtmellec, M. Brunel, D. Allano, D. Lebrun, and A. J. Janssen, "Application of the fractional Fourier transformation to digital holography recorded by an elliptical, astigmatic Gaussian beam," J. Opt. Soc. Am. A 22, 2569-2577 (2005).

[27] J. Braat, P. Dirksen, and A. J. E. M. Janssen, "Assessment of an extended Nijboer-Zernike approach for the computation of optical point-spread functions," J. Opt. Soc. Am. A 19, 858-870 (2002).

[28] W. Xu, M. H. Jericho, I. A. Meinertzhagen, and H. J. Kreuzer, “Digital in-line holography for biological applications," PNAS 98, 11301-11305 (2001).

[29] S. Coëtmellec, D. Lebrun, and C. Özkul, "Characterization of diffraction patterns directly from in-line holograms with the fractional Fourier transform," Appl. Opt. 41, 312-319 (2002).

[30] V. Namias, "The fractional order Fourier transform and its application to quantum mechanics," IMA J. Appl. Math. 25, 241-265 (1980).

[31] A. C. McBride, and F. H. Kerr, "On Namias's fractional Fourier transforms," IMA J. Appl. Math. 39, 159-175 (1987).

[32] A. W. Lohmann, "Image rotation, Wigner rotation, and the fractional Fourier transform," J. Opt. Soc. Am. A 10, 2181-2186 (1993).

[33] N. Verrier, S. Coëtmellec, M. Brunel, and D. Lebrun, “Digital in-line holography in thick optical systems: application to visualization in pipes," Appl. Opt. 47, 4147-4157 (2008).

[34] S. Coëtmellec, C. Remacha, M. Brunel, D. Lebrun, and A. J. E. M. Janssen, "Digital in-line holography with a spatially partially coherent beam," J. Europ. 0pt. Soc. Rap. Public. 6, 11060 (2011).

[35] M. Leclercq, and P. Picart, "Digital Fresnel holography beyond the Shannon limits," Opt. Express 20, 18303-18312 (2012).
[36] X. Wu, S. Meunier-Guttin-Cluzel, Y. Wu, S. Saengkaew, D. Lebrun, M. Brunel, L. Chen, et al., "Holography and micro-holography of particle fields: a numerical standard," Opt. Commun. 285, 3013-3020 (2012)

[37] F. Lamadie, L. Bruel, and M. Himbert, "Digital holographic measurement of liquid-liquid two-phase flows," Opt. Laser. Eng. 50, 1716-1725 (2012).

[38] F. Slimani, G. Grehan, G. Gouesbet, and D. Allano, "Near-field Lorenz-Mie theory and its application to microholography," Appl. Opt. 23, 4140-4148 (1984).

[39] J. Goodman, Introduction To Fourier Optics (Roberts and Company Publishers, Englewood, 2005).

[40] R. M. Aarts, and A. J. Janssen, "On-axis and far-field sound radiation from resilient flat and dome-shaped radiators," J. Acoust. Soc. Am. 125, 1444-1455 (2009).

[41] J. J. M. Braat, P. Dirksen, A. J. E. M. Janssen, and A. S. van de Nes, "Extended Nijboer-Zernike representation of the vector field in the focal region of an aberrated high-aperture optical system," J. Opt. Soc. Am. A 20, 2281-2292 (2003).

[42] M. J. Bastiaans, and T. Alieva, "Signal representation on the angular Poincaré sphere, based on second-order moments," J. Opt. Soc. Am. A 27, 918-927 (2010).

[43] M. Abramowitz, and I. A. Stegun, Handbook of Mathematical Functions (Dover, New York, 1970).

[44] W. J. Tango, "The circle polynomials of Zernike and their application in optics," Appl. Phys. 13, 327-332 (1977).

[45] H. Takahasi, and M. Mori, "Double exponential formulas for numerical integration," Publ. RIMS, Kyoto Univ. 9, 721-741 (1974).

[46] M. Muhammad, and M. Mori, "Double exponential formulas for numerical indefinite integration," J. Comput. Appl. Math. 161, 431-448 (2003).

[47] M. Mori, "Discovery of the double exponential transformation and its developments," Publ. RIMS, Kyoto Univ. 41, 897-935 (2005)

[48] C. Van Der Avoort, J. J. M. Braat, P. Dirksen, A. J. E. M. Janssen, "Aberration retrieval from the intensity point-spread function in the focal region using the extended Nijboer-Zernike approach," J. Mod. Optic. 52, 1695-1728 (2005). 